\title{
Pterostilbene Promotes Mean Lifespan in Both Male and Female Drosophila Melanogaster Modulating Different Proteins in the Two Sexes
}

\author{
Daniela Beghelli $\mathbb{D}^{1}{ }^{1}$ Lorenzo Zallocco, ${ }^{2}$ Maria Cristina Barbalace, ${ }^{3}$ Simona Paglia, ${ }^{4}$ \\ Silvia Strocchi, ${ }^{5}$ Ilenia Cirilli, ${ }^{6}$ Valeria Marzano, ${ }^{7}$ Lorenza Putignani, ${ }^{8}$ Giulio Lupidi, ${ }^{6}$ \\ Silvana Hrelia $\mathbb{D}^{3},{ }^{3}$ Laura Giusti, ${ }^{6}$ and Cristina Angeloni $\mathbb{C}^{3}$ \\ ${ }^{1}$ School of Biosciences and Veterinary Medicine, University of Camerino, Via Gentile III da Varano, 62032 Camerino (MC), Italy \\ ${ }^{2}$ Department of Pharmacy, University of Pisa, 56126 Pisa, Italy \\ ${ }^{3}$ Department for Life Quality Studies, Alma Mater Studiorum, University of Bologna, Corso d'Augusto 237, 47921 Rimini (RN), Italy \\ ${ }^{4}$ Department of Pharmacy and Biotechnology, University of Bologna, Via Selmi 3, 40126 Bologna (BO), Italy \\ ${ }^{5}$ Translational Research Lab-Santa Maria Nuova Hospital-IRCCS, Viale Umberto I 50, 42123 Reggio Emilia, Italy \\ ${ }^{6}$ School of Pharmacy, University of Camerino, Via Gentile III da Varano, 62032 Camerino (MC), Italy \\ ${ }^{7}$ Multimodal Laboratory Medicine Research Area, Unit of Human Microbiome, Bambino Gesù Children's Hospital, IRCCS, Viale di \\ San Paolo 15, 00146 Rome, Italy \\ ${ }^{8}$ Department of Diagnostics and Laboratory Medicine, Unit of Microbiomics and Multimodal Laboratory Medicine Research Area, \\ Unit of Human Microbiome, Bambino Gesù Children's Hospital, IRCCS, Viale di San Paolo 15, 00146 Rome, Italy
}

Correspondence should be addressed to Cristina Angeloni; cristina.angeloni@unicam.it

Received 5 October 2021; Revised 2 February 2022; Accepted 3 February 2022; Published 16 February 2022

Academic Editor: Cristina Cosentino

Copyright (C) 2022 Daniela Beghelli et al. This is an open access article distributed under the Creative Commons Attribution License, which permits unrestricted use, distribution, and reproduction in any medium, provided the original work is properly cited.

\begin{abstract}
Aging is a multifactorial phenomenon characterized by degenerative processes closely connected to oxidative damage and chronic inflammation. Recently, many studies have shown that natural bioactive compounds are useful in delaying the aging process. In this work, we studied the effects of an in vivo supplementation of the stilbenoid pterostilbene on lifespan extension in Drosophila melanogaster. We found that the average lifespan of flies of both sexes was increased by pterostilbene supplementation with a higher effect in females. The expression of longevity related genes (Sir2, Foxo, and Notch) was increased in both sexes but with different patterns. Pterostilbene counteracted oxidative stress induced by ethanol and paraquat and up-regulated the antioxidant enzymes Ho e Trxr-1 in male but not in female flies. On the other hand, pterostilbene decreased the inflammatory mediators dome and egr only in female flies. Proteomic analysis revealed that pterostilbene modulates 113 proteins in male flies and only 9 in females. Only one of these proteins was modulated by pterostilbene in both sexes: vacuolar H[+] ATPase $68 \mathrm{kDa}$ subunit 2 (Vha68-2) that was strongly down-regulated. These findings suggest a potential role of pterostilbene in increasing lifespan both in male and female flies by mechanisms that seem to be different in the two sexes, highlighting the need to conduct nutraceutical supplementation studies on males and females separately in order to give more reliable results.
\end{abstract}

\section{Introduction}

Aging is a natural physiological process triggered by different molecular pathways and biochemical events that are promoted by both environmental and genetic factors. Aging is characterized by a time-dependent decline of functional capabilities and impaired stress resistance, that damage biomolecules compromising cellular homeostasis. The factors involved in aging are commonly referred to as the "hallmarks of aging". Among them, oxidative stress and inflammation have been widely investigated. In 1956 Denham Harman [1] proposed"The Free Radical Theory of Aging" 


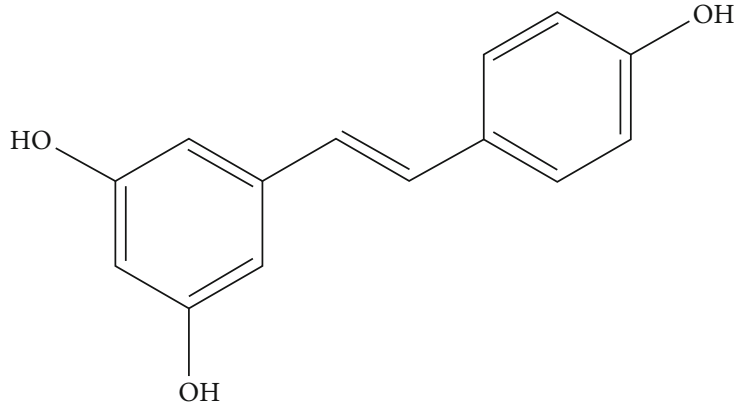

(a)

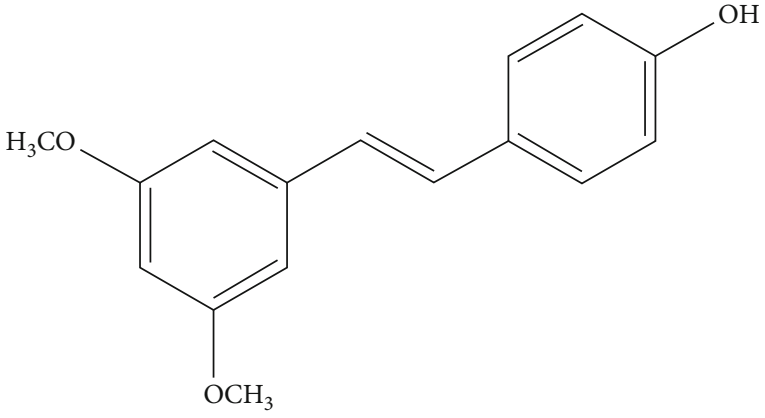

(b)

Figure 1: Chemical structure of A) resveratrol and B) pterostilbene.

that has been updated in recent years to include the suggestion of a central role for reactive oxygen species (ROS) produced by mitochondria [2]. On the other hand, the term "inflammaging" has been created to indicate the significant contribution of low-grade, systemic inflammation to normal aging [3].

The findings that the modification of environmental factors, such as diet, can increase lifespan, make natural dietary compounds extraordinary potential tools in the major healthcare challenge of delaying aging. Over the past 20 years, many studies have suggested that dietary polyphenols may exert beneficial effects as anti-aging compounds through the modulation of the hallmarks of aging, including inflammation, oxidative damage, cell senescence and telomere attrition [4-8]. Among the most prominent polyphenols, the stilbene resveratrol (5-[(E)-2-(4-hydroxyphenyl)ethenyl]benzene-1,3-diol) (Figure 1(a)) has gained widespread attention due to its ability to extend the lifespan of yeast, worms, and flies, and its ability to protect against age-related diseases such as cancer, Alzheimer's disease, and diabetes in mammals $[9,10]$.

Recently, pterostilbene (PTS) (Figure 1(b)) a dimethoxylated ether resveratrol derivative, has been gaining more and more attention thanks to its greater in vivo bioavailability and higher potential in modulating cognition and cellular stress with respect to resveratrol [11]. Both stilbenoids represent a group of natural phenolic compounds present in different types of plants and fruits such as grapes, tree wood, Pterocarpus marsupium, heartwood of sandalwood, leaves of Vitis vinifera and blueberries $[12,13]$.

Resveratrol has been more extensively characterized in terms of bioavailability and pharmacokinetics in human and animal studies compared to PTS. [9, 14-17]. What is known about PTS is that its structure, which differs from that of resveratrol by the presence of two methoxy ($\left.\mathrm{OCH}_{3}\right)$ instead of two hydoxy $(-\mathrm{OH})$ groups, may overcome the pharmacologic efficacy limitations observed in resveratrol (due to its poor absorption and rapid first-pass metabolism). When PTS is orally administered, it shows higher bioavailability ( $80 \%$ vs $20 \%$ of resveratrol), hepatic stability and total plasma levels when compare to resveratrol [18, 19]. Riche et al. [20] showed that PTS in a dose up to $250 \mathrm{mg}$ per day is safe for humans and, thanks to its dimethoxy structure, that creates a favorable lipophilicity, has a higher membrane permeability and biological potency [21]. Furthermore, glucuronidation and sulfation processes are restricted by the methylation of the phenolic hydroxyl in PTS thus providing fewer conjugating sites compared to resveratrol and resulting in better metabolic stability.

In 2003, Howitz and colleagues [22] identified resveratrol as a potent activator of Sir2, the mammalian Sirt1 ortholog, capable of mimicking the effects of caloric restriction (CR) and regulating longevity in lower organisms such as worms and yeasts, while in Drosophila melanogaster there are contradictory results [23-25]. Besides $\mathrm{NAD}^{+}$dependent histone deacetylase Sir2 [26], that controls enzymes regulating a large number of cellular pathways [27], there are other genes associated with longevity in Drosophila. Reduction in the activity of the nutrient-sensing insulin/IGF Signaling (IIS) pathway and the associated Target of Rapamycin (TOR) pathway results in one of the best understood interventions to extend flies lifespan [26]. The IIS pathway, in turn, intersects with a variety of other pathways that impact longevity such as the stress-responsive Jun-N-terminal kinase pathway, or the pathways that promote protein homeostasis and activate the mitochondrial unfolded protein response. Lifespan is also regulated by other mechanisms such as the loss of the mitochondrial co-transporter I'm Not Dead Yet (INDY) and the modulation of the mitochondrial electron transport chain [26].

To the best of our knowledge, there is no evidence that shows a direct effect of PTS on lifespan extension in vivo. Therefore, the present work sets out to study the potential role of PTS on longevity, oxidative stress and inflammation in D. melanogaster taking into consideration sex differences. We also carried out proteomic analysis on the whole Drosophila body, to have a deeper insight in the potential different effects of PTS supplementation on male and female flies.

\section{Materials and Methods}

2.1. Fly Strain, Rearing and Supplementation. The Drosophila melanogaster Canton S strain was a kind gift of Prof. Daniela Grifoni (University of l'Aquila, Italy). Flies were supplied with Formula 4-24 ${ }^{\circledR}$ media (Carolina Biological, Burlington, NC, USA) and maintained at constant temperature $\left(21^{\circ} \mathrm{C}\right)$ and humidity $(60 \%)$ with a $12 / 12 \mathrm{~h}$ light-dark cycle. The diet contained: oat flour, soy flour, wheat flour, 
other starches, dibasic calcium phosphate, calcium carbonate, citric acid, niocinamide, riboflavin, sodium chloride, sodium iron pyrophosphate, sucrose, thiamine, mononitrate, brewer's yeast, emulsifier preservatives, mold inhibitor, food coloring [28]. Yeast pellets (Saccharomyces cerevisiae) were added to each tube after diet hydration. After eclosion, males and females were synchronized as reported in [29] and allowed to mate freely for two days before separating them according to sex [30]. PTS (Sigma-Aldrich s.r.l., Milan, Italy) was dissolved in DMSO (0.1\%) and 50, 100, and $200 \mathrm{mM}$ stocks were prepared and kept at $-20^{\circ} \mathrm{C}$ until use. For supplementation, PTS was dissolved at different concentrations $(50,100$ or $200 \mu \mathrm{M})$ in the water used to soak $1.0 \mathrm{~g}$ of diet. A total of 20 flies were placed in each vial. The water used to prepare the control food contained $0.1 \%$ of DMSO.

2.2. Longevity Assay. Adult flies of both sexes were collected under FlyNap (Carolina Biological) anesthesia. A total of 800 male and 800 female fruit flies were randomly divided into 4 groups: CTR (control), PTS $50(50 \mu \mathrm{M}$ PTS), PTS $100(100 \mu \mathrm{M}$ PTS $)$, and PTS $200(200 \mu \mathrm{M}$ PTS). Every $2-3$ days, the flies were transferred into vials containing fresh food and the number of living flies was counted. This was repeated until all flies died. Kaplan-Meier survival curves were generated for lifespan assessment.

2.3. Measurement of Body Weights. Body weights of male and female flies were recorded on days 15, 30, 45 and 60. Briefly, 20 flies in each group were anesthetized by FlyNap (Carolina Biological) and then weighed on a balance. The mean body weights of the flies in each group were calculated.

2.4. CApillary FEeder (CAFE) Assay. Food intake was measured using the capillary feeder method (CAFE) as reported in [31] with minor modifications. Flies were held in shortened culturing vials of $7 \mathrm{~cm}$ in length. Cotton balls, soaked with $500 \mu \mathrm{L}$ of water, were inserted at the bottom of tubes for humidity, the tubes were then parafilmed to reduce evaporation and keep a high level of humidity inside the vials. Microcapillary tubes were inserted into the tubes through a $200 \mu \mathrm{L}$ pipette tip in the foam plug. Four microcapillary tubes were used per vial and filled with $100 \mu \mathrm{M}$ PTS (PTS) or $0.1 \%$ DMSO diluted in $2.5 \%$ sucrose (CTRL). The assay was performed on flies never supplemented ( 3 days old male and female flies) or on flies supplemented with PTS or $0.1 \%$ DMSO for 15 days. At each time point 2 groups for sex were considered: PTS and CTRL. On the day of the experiment, flies were weighted, starved for two hours, and then separated into CAFE vials, with eight flies for vial (40 flies/sex/ condition; $\mathrm{n}=80$ flies). To account for evaporation of the liquid food, three vials were set up with feeding capillaries but without flies. Fly consumption was evaluated after four hours measuring the amount of liquid consumed from the microcapillary tube (in $\mathrm{mm}$ ) as described by Fiocca et al. [32] and data were reported as $\mu \mathrm{L} / \mathrm{mg}$ of fly.

2.5. Paraquat Toxicity. Oxidative stress resistance was measured using paraquat $\left(1,1^{\prime}\right.$-dimethyl-4,4'-bi-pyridinium dichloride, Sigma-Aldrich s.r.l.) as stress inducer. Briefly, 15 days-old flies were subjected to starvation for 2 hours then transferred to parafilm-sealed vials (ten flies per vial) containing capillaries filled with $2.5 \mathrm{mM}$ paraquat prepared with $2.5 \%$ sucrose solution. For each condition, 50 flies per sex were tested. Mortality was scored every 6 hours until all flies died and expressed as percentage of survival.

2.6. Ethanol Toxicity. Ethanol toxicity was evaluated as reported by Niveditha [33] with some modifications. Briefly, 15 days-old flies (supplemented and CTR) were first subjected to starvation for $2 \mathrm{~h}$ then transferred to parafilmsealed vials (ten flies per vial) containing Whatman filter paper discs (diameter $2 \mathrm{~cm}$ ) soaked with 2.5\% sucrose solution and $17.5 \%$, ethanol (Carlo Erba, Milano, Italy). Mortality was scored every 2 hours until all flies died and expressed as percentage of survival. For each condition, 50 flies per sex were tested.

2.7. DCFH-DA Assay. ROS content was evaluated using $2^{\prime}$ $-7^{\prime}$-dichlorodihydrofluorescein diacetate (DCFH-DA). Groups of 25 flies were mechanically homogenized in $1 \mathrm{~mL}$ of $0.1 \mathrm{M}$ PBS. The homogenate was centrifuged at $6,000 \mathrm{rpm}$ for $10 \mathrm{~min}$ and $200 \mu \mathrm{L}$ of supernatant was incubated with $50 \mu \mathrm{M}$ DCFH-DA (Sigma-Aldrich s.r.l.) at $37^{\circ} \mathrm{C}$. The fluorescence was measured each 10 minutes in a FLUOstar Omega plate reader (BMG Labtech, Ortenberg, Germany) using ex/em wavelength of $485 / 520 \mathrm{~nm}$. ROS levels were quantified as increase in DCF fluorescence through the time normalized by protein and expressed as increase $/ \mathrm{min} / \mu \mathrm{g}$ protein. The protein concentration in the homogenate was quantified by Bradford method using bovine serum albumin (Sigma-Aldrich s.r.l) as standard. Each condition was the results of six replicates and was expressed as mean \pm SEM.

2.8. Total Antioxidant Capacity. To assess the total antioxidant capacity of flies, the 2, $2^{\prime}$-azino-bis (3-ethylbenzothiazoline-6-sulphonic acid, Sigma-Aldrich s.r.l.) (ABTS) assay, as reported by Re et al. [34] and modified for application to a 96-well microplate, was used.

Flies were mechanically homogenized in $1 \mathrm{~mL}$ of $0.1 \mathrm{M}$ PBS on ice. The homogenate was centrifuged at $6,000 \mathrm{rpm}$ for $10 \mathrm{~min}$ and the supernatant was used for the assay.

The $\mathrm{ABTS}^{\bullet+}$ solution was freshly prepared by the oxidation of ABTS $(10 \mathrm{mg})$ by $\mathrm{MnO}_{2}(0.75 \mathrm{~g})$ in the presence of water $(4 \mathrm{~mL})$, followed by $30 \mathrm{~min}$ at room temperature. The working solution was obtained by diluting the previous mixture with $\mathrm{H}_{2} \mathrm{O}$ to have an absorbance around 1 at $734 \mathrm{~nm}$.

For the assay, $200 \mu \mathrm{L}$ of the working solution was added to $50 \mu \mathrm{L}$ aliquot of progressively diluted samples and Trolox as a standard. The absorbance of each well was measured after $15 \mathrm{~min}$ incubation at $734 \mathrm{~nm}$.

The protein concentration in the homogenates was quantified by Bradford method using bovine serum albumin as standard. Each condition was the results of six replicates and was expressed as mean \pm SEM. The total antioxidant capacity of flies was compared to Trolox used as positive control and expressed as mg of Trolox-equivalent antioxidant capacity for $\mathrm{mg}$ of homogenate protein (TEAC). 
TABLE 1: List of primers for real-time PCR.

\begin{tabular}{lrc}
\hline Gene & \multicolumn{1}{c}{ 5'-Forward-3' } & 5'-Reverse-3' \\
\hline Sir2 & CATTATGCCGCATTTCGCCA & GAAGGTGTTCACTGAGGCCA \\
Foxo & AGGCTGACCCACACAGATAAC & GGCTCCACAAAGTTTCGGG \\
Notch & CGCTTCCTGCACAAGTGTC & GCGCAGTAGGTTTTGCCATT \\
Ho & ATGTCAGCGAGCGAAGAAACA & TGGCTTTACGCAACTCCTTTG \\
Trxr-1 & TGGATCTGCGCGACAAGAAAG & GAAGGTCTGGGCGGTGATTG \\
Dome & GGCAGCTTCTATGTCTACTC & GTTGGACTCCACCTTGATG \\
Egr & GAAATCACACAGAGCTTCAG & AAGAAGAGATTCACCTTTGC \\
RPL32 & GCCCACCGGATTCAAGAAGT & CTTGCGCTTCTTGGAGGAGA \\
\hline
\end{tabular}

2.9. RNA Extraction. RNA was extracted from the whole bodies of flies using RNeasy Mini Kit (QIAGEN GmbH, Hilden, Germany). NanoVue Spectrophotometer (GE Healthcare, Milano, Italy) was used to measure the yield and purity of the RNA. Only samples with ratios A260/ A280 $>1.8$ were used.

2.10. Analysis of mRNA Levels by Reverse Transcriptase Polymerase Chain Reaction. For each sample, $1 \mu \mathrm{g}$ of total RNA was reverse transcribed to obtain cDNA using iScript cDNA Synthesis Kit (Bio-Rad Laboratories, Hercules, CA, USA) following the manufacturer's instructions. The subsequent polymerase chain reaction (PCR) was performed in a total volume of $10 \mu \mathrm{L}$ containing $2 \mu \mathrm{L}$ of $\mathrm{dH}_{2} \mathrm{O}$ RNAsi free, $2.5 \mu \mathrm{L}(12.5 \mathrm{ng})$ of $\mathrm{cDNA}, 5 \mu \mathrm{L}$ SsoAdvanced Universal SYBR Green Supermix (Bio-Rad Laboratories), and $0.5 \mu \mathrm{L}$ $(500 \mathrm{nM})$ of each primer. The primers used were purchased from Sigma-Aldrich s.r.l. and they are reported in Table 1; RPL32 was used as reference gene.

2.11. Proteomic Analysis. For proteomic analysis, proteins were extracted from total body of male and female flies supplemented with $100 \mu \mathrm{M}$ PTS for 15 days using $8 \mathrm{M}$ urea, $2 \mathrm{M}$ thiourea, $4 \%$ CHAPS and $60 \mathrm{mM}$ dithiothreitol (DTT) extraction solution. Briefly, bodies of flies were resuspended in extraction solution using a microtube pestle and sonicated 1 min for 5 times in an ultrasonic bath. After incubation for $1 \mathrm{~h}$ at room temperature, samples were centrifuged at 16,000 $\mathrm{x} \mathrm{g}$ for $10 \mathrm{~min}$ to remove undissolved material. Protein concentration was determined using the Pierce Protein Assay (Thermo Fisher Scientific, Waltham, MA, USA) and bovine serum albumin was used as standard. Two-dimensional gel electrophoresis (2DE) was carried out as previously described [35]. Briefly, $200 \mu \mathrm{g}$ of proteins were filled up to $350 \mu \mathrm{L}$ in rehydration solution. Isoelectrofocusing (IEF) was performed using $18 \mathrm{~cm}$ Immobiline Dry Strips (GE Health Care Europe, Uppsala, Sweden) with a nonlinear $\mathrm{pH}$ 3-10 gradient. IEF was carried on the Ettan IPGphor Cup Loading Manifold (GE Healthcare). After IEF, the strips were equilibrated during two steps of $15 \mathrm{~min}$ each in equilibration buffer (0.05 M Tris, $6 \mathrm{M}$ urea, $2 \%$ SDS, $20 \%$ glycerol) with $1 \%$ DTT in the first incubation or $2.5 \%$ iodoacetamide in the second one. The subsequent electrophoresis (Sodium Dodecyl Sulphate-Polyacrylamide Gel Electrophoresis; SDS-PAGE) was carried out by transferring the proteins to $12 \%$ polyacryl- amide, running at $16 \mathrm{~mA}$ per gel and $10^{\circ} \mathrm{C}$ for about $16 \mathrm{~h}$, using the Protean ${ }^{\circledR}$ Plus Dodeca Cell (Bio-Rad). The gels were stained with Ruthenium II tris (bathophenanthroline disulfonate) tetrasodium salt (Cyanagen, Bologna, Italy) (RuBP). ImageQuant LAS4010 (GE Health Care) was used for the acquisition of images. The analysis of images was performed using Same Spot (v4.1, TotalLab, Newcastle Upon Tyne, UK) software.

Gel spots were excised and in-gel digested [36]. Peptides were lyophilized and resuspended in $2 \%$ acetonitrile (ACN), $0.1 \%$ formic acid (FA) and $97.9 \%$ water.

2.12. Mass Spectrometry Analysis. NanoLiquid Chromatography coupled tandem mass spectrometry analysis (nLCESI-MS/MS) was achieved on an analytical platform comprising an UltiMate3000 RSLCnano System interfaced with an Orbitrap Fusion Tribrid mass spectrometer through a nanoESI source (EASY-Spray NG) (Thermo Fisher Scientific, Milan, Italy) operating in positive ion mode, as already described [37] with minor modifications. After trapping and desalting on a micro-precolumn, a 15 min linear gradient starting from $95 \%$ solution $\mathrm{A}(0.1 \% \mathrm{FA}$ in water) to $25 \%$ solution B (0.1\% FA in ACN) allowed peptides' separation on an EASY-Spray PepMap RSLC C18 column $(2 \mu \mathrm{m}$ particle size, $100 \AA$ pore size, $75 \mu \mathrm{m}$ i.d. x $25 \mathrm{~cm}$ length, Thermo Fisher Scientific) for a total run of $40 \mathrm{~min}$.

Precursor ions were recorded by the Orbitrap detector and fragments (MS/MS) ions by the Ion Trap at rapid scan rate. Twenty most abundant multiple-charged $\left(2^{+}-7^{+}\right)$precursor ions, detected within the range of $275-1,750 \mathrm{~m} / z$, were subjected to fragmentation by Collision-Induced Dissociation (CID) with dynamic exclusion of $45 \mathrm{~s}$ and using $35 \%$ normalized collision energy. The signal intensity threshold for MS/MS was set to $5 \times 10^{3}$. Proteome Discoverer software (version 2.4, Thermo Fisher Scientific) was used to process the raw data searching the fruit fly UniProtKB reference proteome (Drosophila melanogaster database, ID: UP000000803, release: 2021_01, 22,117 proteins) to which 39 common contaminant sequence entries were appended. Protein identification search parameters were set as follows: chosen enzyme: trypsin with a maximum of 1 missed cleavage per peptide; variable modification: oxidation of methionine and acetylation of lysines at protein $\mathrm{N}$-terminus; static modification: carbamidomethylation of cysteine; mass tolerance of precursor: $10 \mathrm{ppm}$; fragment 

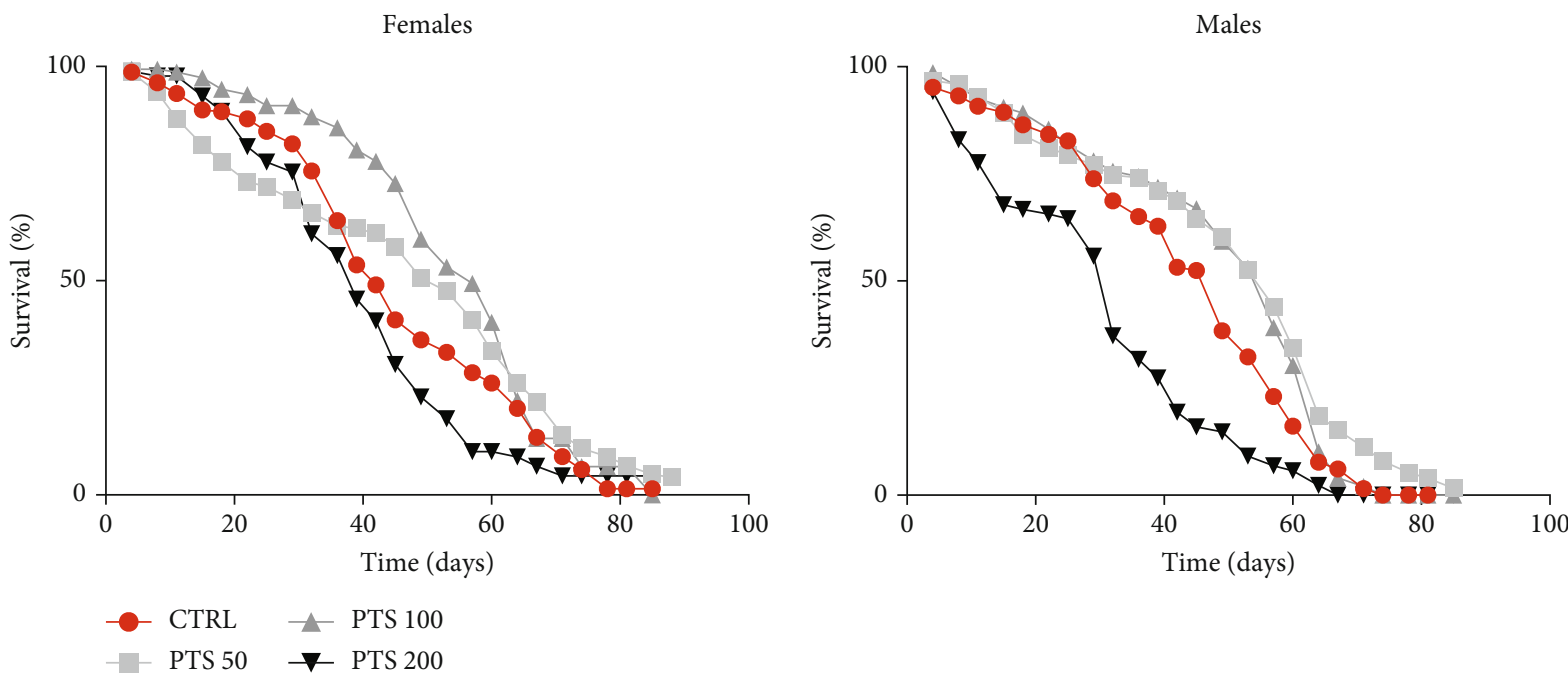

FIgURE 2: Survival assay of adult female and male flies. Flies were supplemented with 50, 100, and $200 \mu \mathrm{M}$ PTS lifelong. Data are presented as percentage of survival of flies as function of time (in days). The Kaplan-Meier test was used to detect the significant differences among the fourth groups of flies.
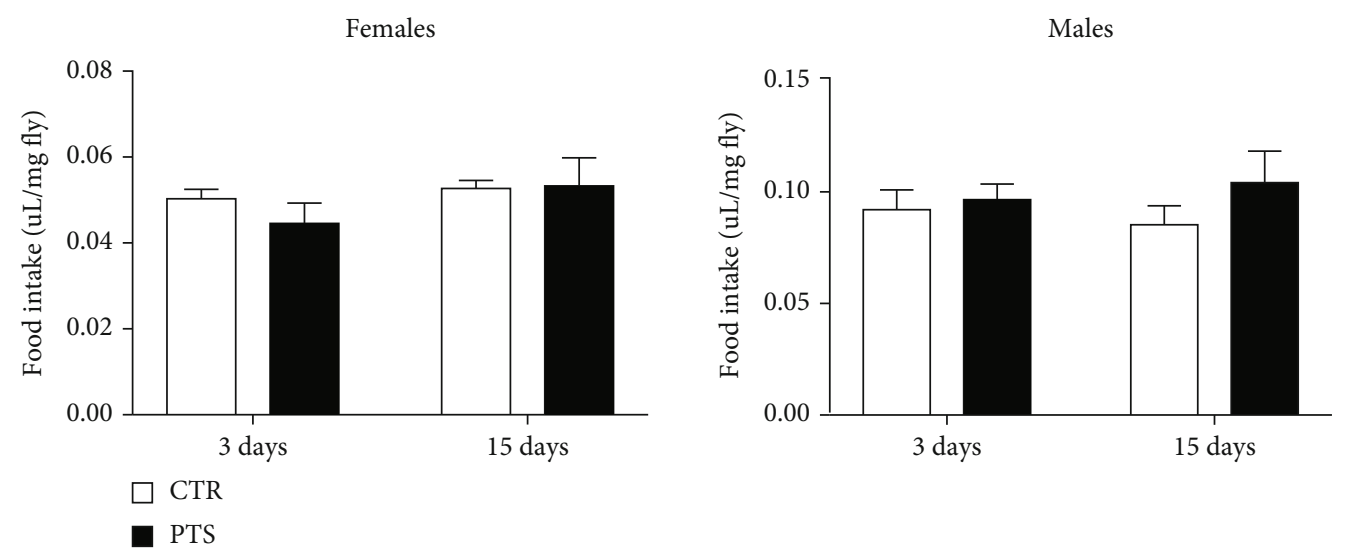

(a)
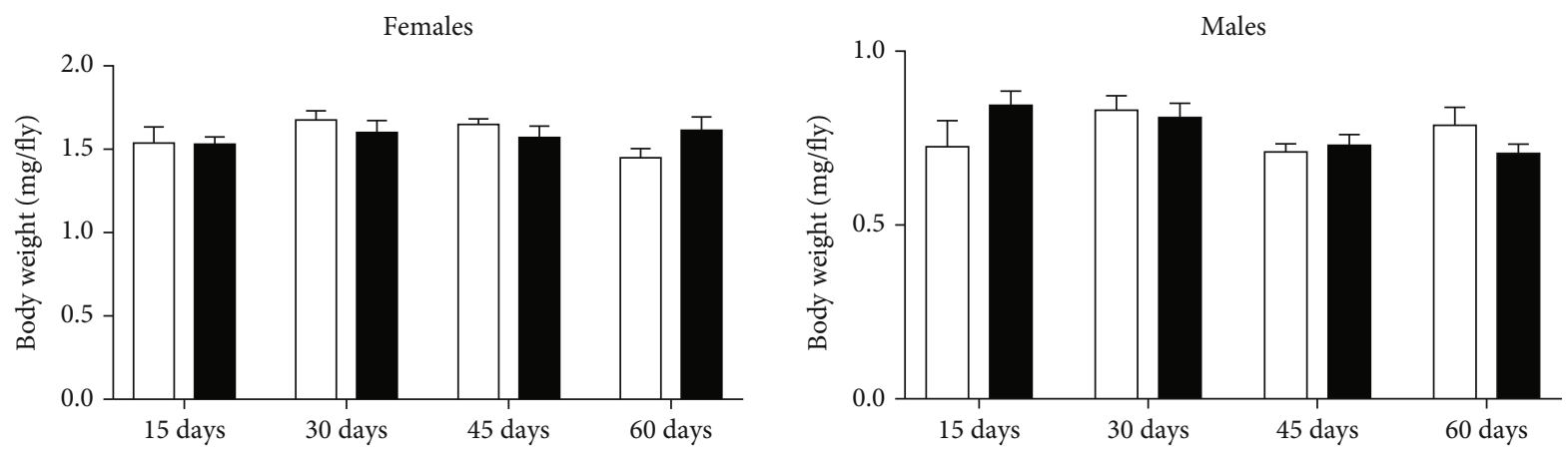

$\square$ CTR

— PTS

(b)

FIgURE 3: Food intake and body weights of D. melanogaster supplemented with PTS. (a). Flies were not supplemented with PTS or supplemented with $100 \mu \mathrm{M}$ PTS for 15 days before CAFE assay. (b). Flies were supplemented with $100 \mu \mathrm{M}$ PTS for 15, 30, 45, and 60 days before body weight measurement. Each bar represents the mean \pm SEM. Data were analyzed by Student's t-test comparing each supplementation to the corresponding control. 

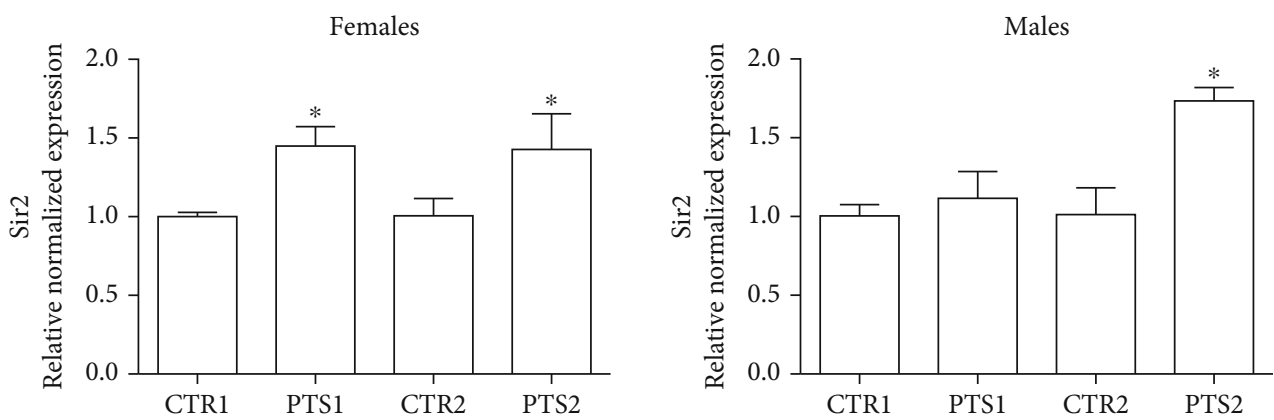

(a)
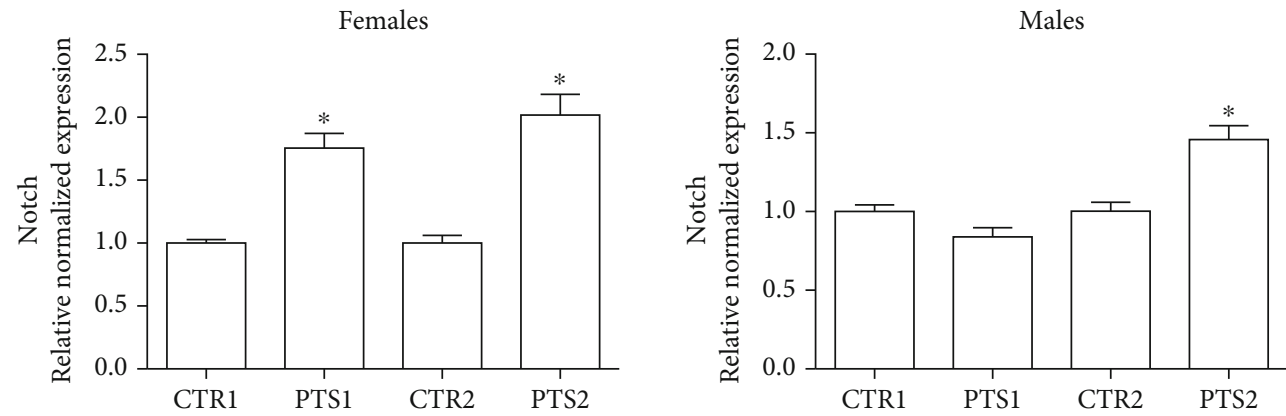

(b)
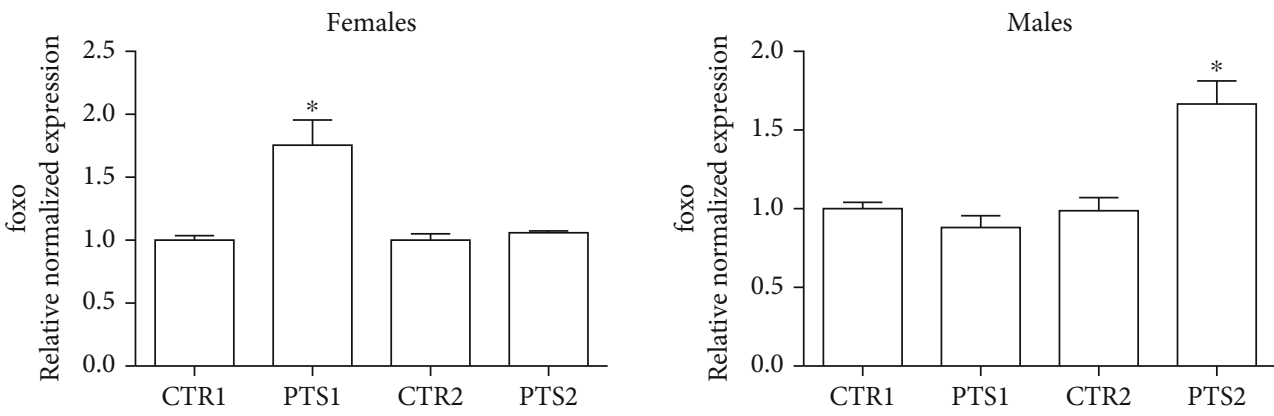

(c)

FIgURE 4: Expression of genes related to longevity in D. melanogaster supplemented with PTS. Flies were supplemented with $100 \mu \mathrm{M}$ PTS for 15 days (PTS1) or 60 days (PTS2). Total RNA was isolated, and the mRNA levels of A) Sir2, B) Notch, and C) foxo were quantified using RT-PCR normalized to RPL32 reference gene as reported in Materials and Methods. Triplicate reactions were performed for each experiment. Each bar represents the mean \pm SEM of three independent experiments. Data relative to 15 days and 60 days were grouped into one graph but analyzed separately by Student's t-test. $* \mathrm{p}<0.05$ with respect to the corresponding controls, CTR1 or CTR2.

mass tolerance: $0.6 \mathrm{Da}$. A threshold of two peptides with high confidence peptide-spectrum matches was applied for protein identification.

2.13. Statistical Analysis. Each experiment was performed at least three times, and all values are represented as means \pm SEM. Student's t-test or one-way ANOVA were used to compare differences among groups followed by Bonferroni's test (Prism 5, GraphPad Software, San Diego, CA). Values of $p<0.05$ were considered statistically significant. Survival curves were prepared by Kaplan-Meier survival analysis and analyzed using the OASIS2 software [38]. For proteomic experiments, statistical analysis was based on the normalized volume of each spot calculated by the software. Comparison analysis was performed between PTS-treated and control images in both females and males, using One-way ANOVA.
Spots that exhibited ratio $\geq 1.2$ or $\leq 0.83, \mathrm{p}$-value $<0.05$ and $\mathrm{q}$ value $<0.05$ were taken into consideration for further protein identification. Bioinformatic analysis was carried out using ShinyGO v.0.66 software to obtain interactive plots showing the relationship between enriched pathways whereas heat map was build using NG-CHM GUI 2.20.2 software [39].

\section{Results}

3.1. Effect of PTS on Longevity. To examine the pro-longevity effect of PTS, male and female Canton S flies were reared on standard diet supplemented with different concentrations of PTS $(50,100$, and $200 \mu \mathrm{M})$ lifelong (Figure 2). DMSO vehicle $(0.1 \%)$ has been added to control groups. DMSO did not influence fly longevity as no significant differences have been observed between control groups reared in the presence or 


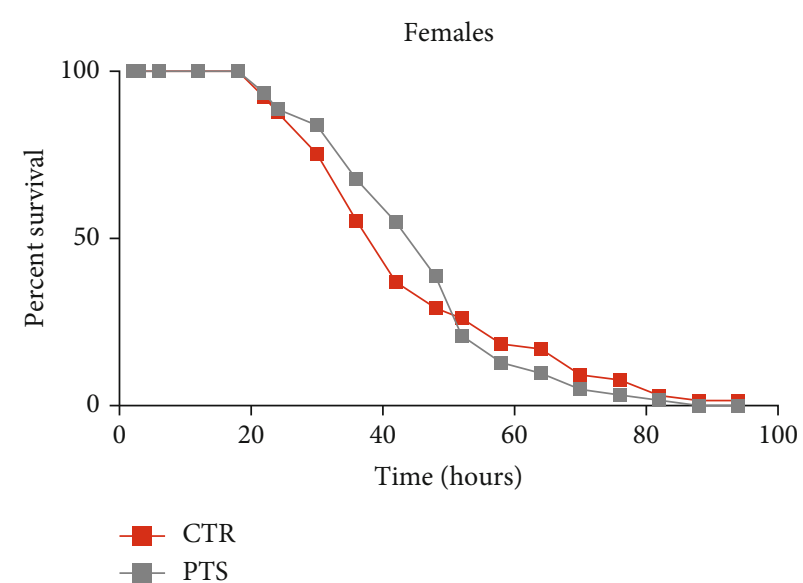

(a)

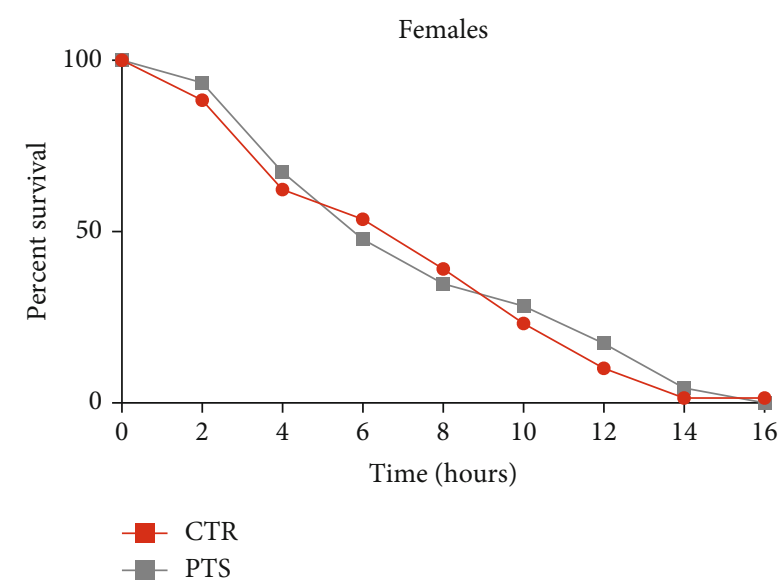

(c)

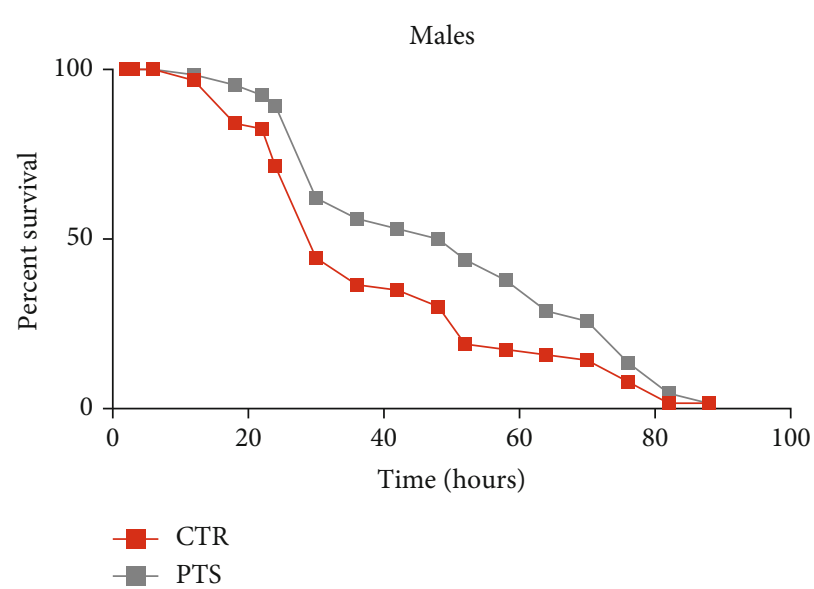

(b)

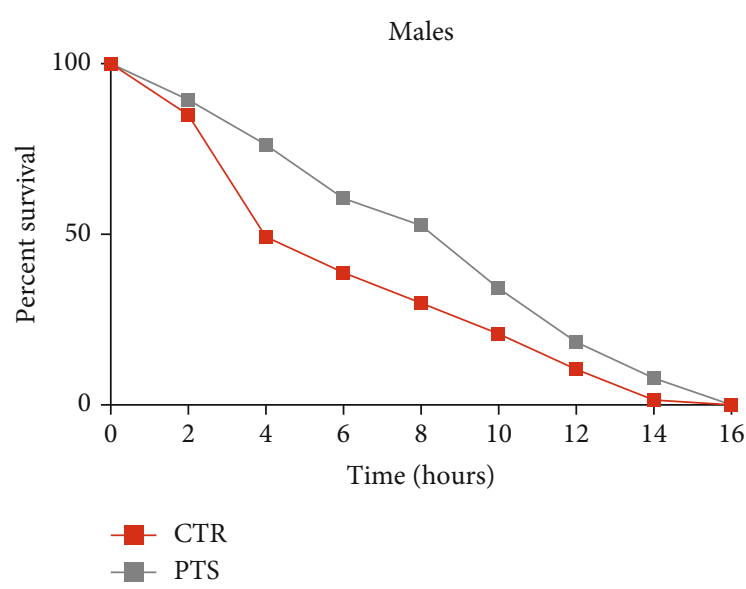

(d)

Figure 5: Survival assay of adult female and male flies after oxidative stress induction. Flies were supplemented with $100 \mu \mathrm{M}$ PTS for 15 days, then were exposed to $2.5 \mathrm{mM}$ Paraquat $(\mathbf{A}, \mathbf{B})$ or $17.5 \%$ ethanol (C, D). Data are presented as percentage survival of flies as function of time (in hours). The Kaplan-Meier test was used to detect the significant differences among the two groups of flies.

absence of $0.1 \%$ DMSO both in male and female flies (data not shown). Moreover, this concentration of DMSO has been used by different authors as control vehicle [40-42]. A significant increase in mean lifespan was observed in $50 \mu \mathrm{M}$ PTS supplemented male flies compared to control flies ( $14 \%$ increase, $\mathrm{p}<0.0034)$, while mean lifespan of female flies treated with $50 \mu \mathrm{M}$ PTS was comparable to that of control flies (Figure 2). $100 \mu \mathrm{M}$ PTS was effective both in male and female flies leading to a significant increase of mean lifespan of $12 \%(\mathrm{p}<0.0196)$ and $20 \%(\mathrm{p}<0.0072)$, respectively. Of note, $200 \mu \mathrm{M}$ PTS had a negative effect in both sexes as it significantly reduced mean lifespan in respect to control flies.

To verify that the improvement of the mean lifespan was due to PTS supplementation itself and not to CR induced by PTS off-flavor, food intake was evaluated by CAFE assay in flies before supplementation and after 15 days of PTS supplementation (Figure 3(a)). Interestingly, PTS did not influence food intake in female and male flies both before and after PTS supplementation. Of note, male flies had a higher food intake in respect to female flies. In particular, PTS supplemented male flies had a significant higher food intake in respect to female PTS supplemented flies both at 3 days and 15 days $(\mathrm{p}<0.0014$ and $\mathrm{p}<0.0341$, respectively). Body weights of flies supplemented with $100 \mu \mathrm{M}$ PTS were recorded on days 15, 30, 45 and 60 (Figure 3(b)) to further confirm that PTS did not modify food intake throughout fly life. No differences in body weights were observed both in female and male flies at any time points, suggesting an equal food intake in control and PTS supplemented groups.

To better clarify, at a molecular level, the effect of PTS on the lifespan of male and female flies, the expression of 3 genes related to longevity were investigated. As $100 \mu \mathrm{M}$ PTS supplementation was effective both in males and females, and in males showed comparable effects to $50 \mu \mathrm{M}$ PTS, $100 \mu \mathrm{M}$ PTS supplementation was used in the following experiments. Flies were supplemented with PTS for 15 days (PTS1) or 60 days (PTS2) and the expression of Sir2, foxo, and Notch was evaluated by RT-PCR (Figure 4).

Sir2, a member of the Sirtuin family of protein acylases, deacetylates lysine residues within many proteins and has been implicated in the extension of longevity in $D$. melanogaster [43]. In Figure 4(a) the expression levels of Sir2 are reported. Sir2 was significantly upregulated in female 

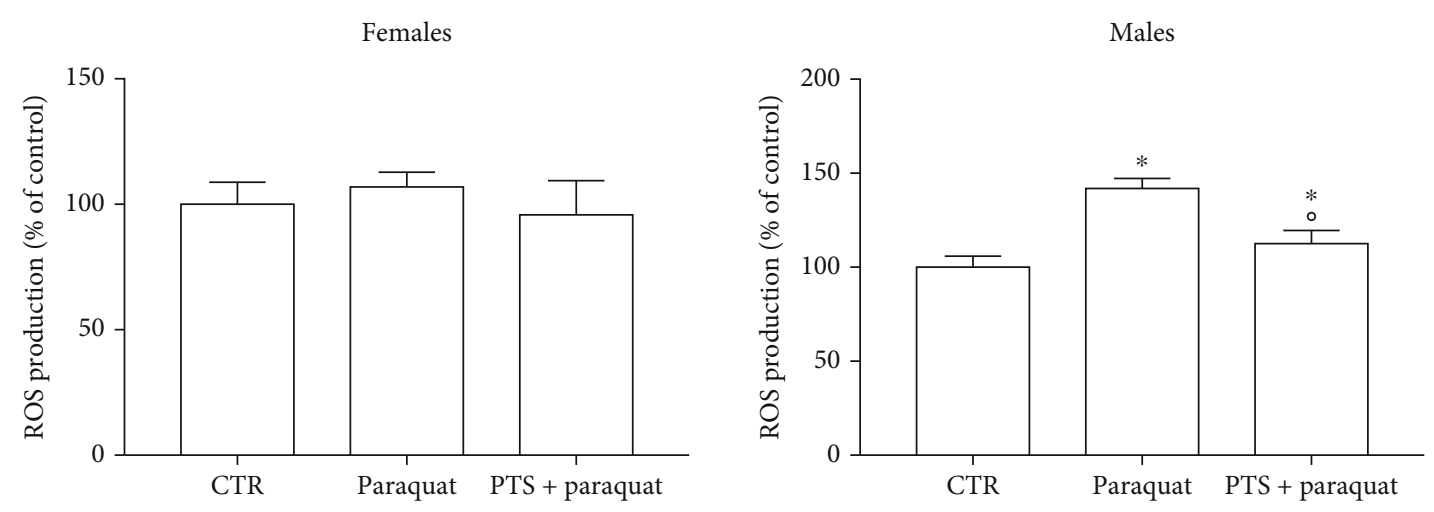

(a)
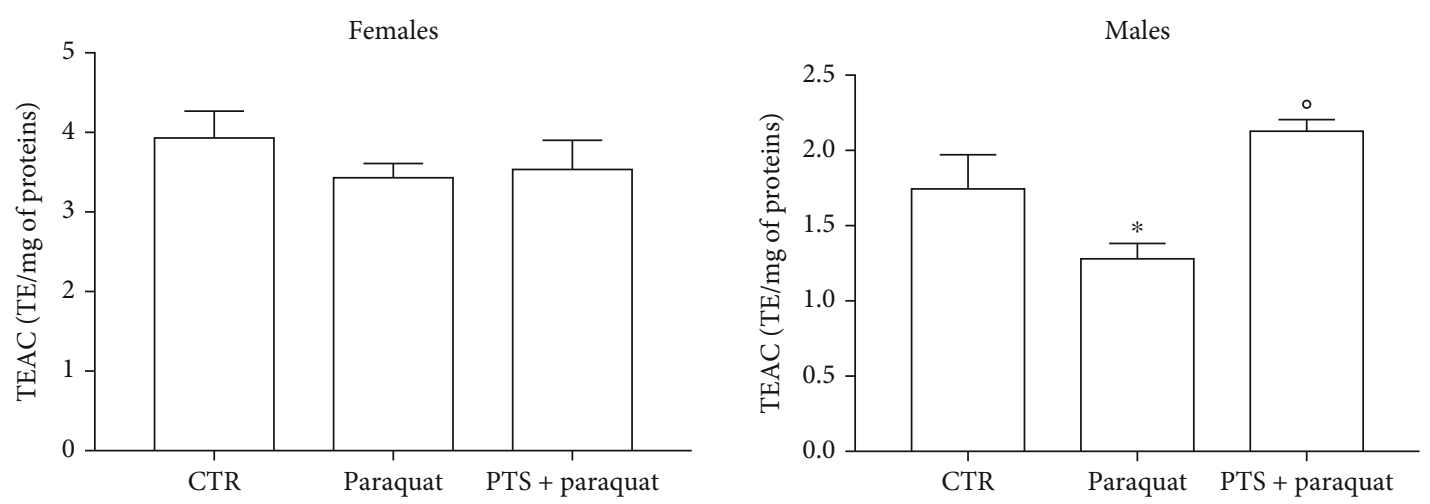

(b)

Figure 6: Redox state of flies supplemented with PTS and exposed paraquat. Flies were supplemented with $100 \mu \mathrm{M}$ PTS for 15 days, then were exposed to $2.5 \mathrm{mM}$ paraquat. A. ROS production was measured with the peroxide-sensitive probe DCFH-DA B. Total radical scavenging capacity was evaluated by ABTS assay. Each value represents mean \pm SD. Data were analyzed by One-way ANOVA followed by Bonferroni's test. $* \mathrm{p}<0.05$ with respect to CTR; ${ }^{\circ} \mathrm{p}<0.05$ with respect to Paraquat.

flies both after 15 days and 60 days, meanwhile in male flies the gene was overexpressed only after 2-month supplementation. As it has been recently demonstrated that Sir2 is a positive regulator of the Notch pathway in Drosophila [44], we also investigate the effect of a short and long term supplementation of PTS on the expression of Notch in male and female flies (Figure 4(b)). Interestingly, the obtained data were perfectly in agreement with the results on Sir2 expression. In fact, in female flies, both the short- and long-term supplementations with PTS were able to up-regulate Notch expression. In male flies only the 2-month supplementation led to a significant Notch over-expression. In D. melanogaster, FOXO, the orthologous of the nematode DAF-16/FoxO and mammalian FOXO3A, has been shown to be a key transcriptional regulator of the insulin pathway that modulates growth and proliferation, and the increase of its activity in certain tissues is sufficient to extend fly lifespan [45]. In our study, PTS triggered a significant foxo up-regulation after 15 days supplementation in female flies and after 2month supplementation in male flies (Figure 4(c)).

3.2. Antioxidant Effect of PTS. To study the antioxidant effect of PTS, flies were supplemented with $100 \mu \mathrm{M}$ PTS for 15 days before the induction of oxidative stress. As we mentioned above, we chose this PTS concentration because, it was the most effective in increasing lifespan in both sexes. Oxidative stress was induced by $2.5 \mathrm{mM}$ paraquat or $17.5 \%$ ethanol exposure in both male and female flies [33, 46-49]. PTS did not show any protective activity against both paraquat and ethanol-induced damage in female flies since the survival curves of control and PTS supplemented flies were comparable (Figure 5(a)-5(c)). On the contrary, $100 \mu \mathrm{M}$ PTS significantly increased the percentage of survival in male flies exposed to both paraquat and ethanol $(\mathrm{p}<0.017$ and $\mathrm{p}<0.0284$, respectively). The redox state of flies supplemented with PTS and exposed to paraquat or ethanol was investigated evaluating both the production of ROS and the total radical scavenging capacity (Figures 6 and 7). In male flies paraquat significantly increased ROS production with respect to control, and PTS administration was able to partially counteract this effect significantly reducing ROS production compared to paraquat but maintaining ROS production to levels significantly higher in respect to controls (Figure 6(a)). These data are strengthened by the results on the antioxidant capacity of male flies, as paraquat significantly reduced the total radical scavenging capacity in respect to controls, meanwhile PTS reverted this effect significantly increasing the endogenous antioxidant levels to value comparable to controls (Figure 6(b)). In female flies paraquat did not modify endogenous ROS production 

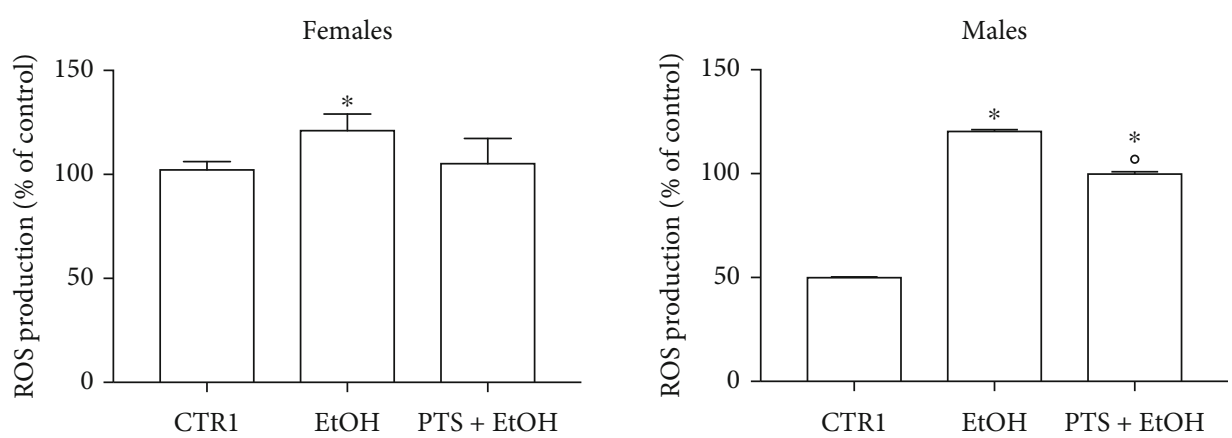

(a)
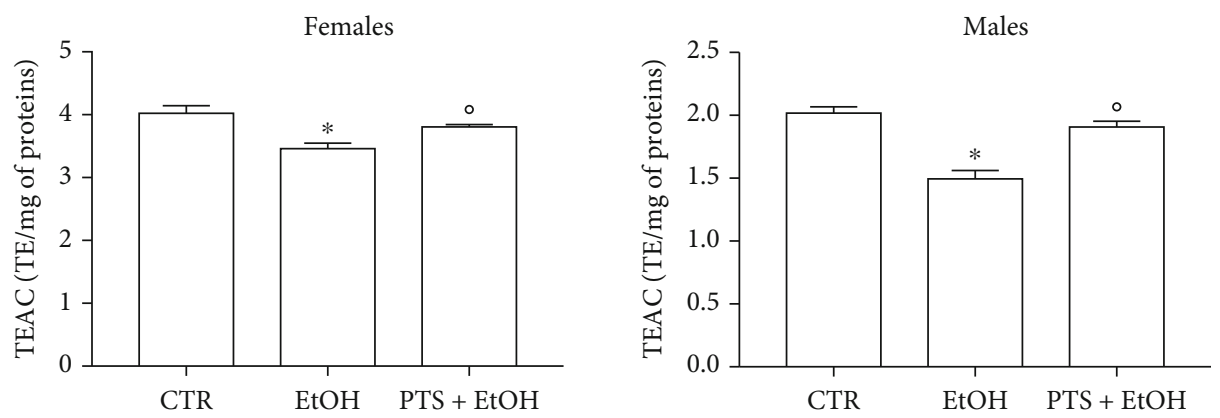

(b)

FIGURE 7: Redox state of flies supplemented with PTS and exposed to ethanol. Flies were supplemented with $100 \mu \mathrm{M}$ PTS for 15 days, then were exposed to $17.5 \%$ ethanol $(\mathrm{EtOH})$ for $4 \mathrm{~h}$. A. ROS production was measured with the peroxide-sensitive probe DCFH-DA B. Total radical scavenging capacity was evaluated by ABTS assay. Each value represents mean \pm SD. Data were analyzed by One-way ANOVA followed by Bonferroni's test. $* \mathrm{p}<0.05$ with respect to CTR; $\mathrm{p}<<0.05$ with respect to EtOH.

(Figure 6(a)). Slight reduction of the total radical scavenging capacity is observable both in females exposed to paraquat and in those pre-treated with PTS even if these results are not statistically significant (Figure 6(b)).

Ethanol significantly and strongly increased ROS production (Figure 7(b)) and decreased the total radical scavenging capacity (Figure 7(c)) in male flies. PTS supplementation significantly decreased ROS levels and increased the total radical scavenging capacity in respect to male flies exposed to ethanol, but only the total radical scavenging capacity reached levels comparable to control flies. In agreement with the data obtained with paraquat, in female flies, ethanol induced a lower increase of ROS levels in respect to male flies. PTS triggered a slight reduction of ROS levels with intermediate values between control and ethanol stressed flies. Total radical scavenging capacity was significantly reduced by ethanol exposure in female flies and PTS was able to significantly increase this value in respect to ethanol treated flies.

To further investigate the antioxidant activity of PTS, the expression of 2 antioxidant genes, heme oxygenase (Ho) and thioredoxin reductase 1 (Trxr-1), was evaluated. Flies were supplemented with PTS for 15 days (PTS1) or 60 days (PTS2) and the expression of Ho and Trxr-1 was measured by RT-PCR (Figure 8 ).

In agreement with the previous data on oxidative stress, PTS did not influence the expression of both the enzymes in female flies. On the other hand, in male flies, PTS supplementation significantly up-regulated Trxr-1 at both supple- mentation times, and triggered Ho over-expression at the second time point.

3.3. Anti-Inflammatory Effect of PTS. One of the most recent theories on aging focuses on the activation of a subclinical, chronic low-grade inflammation that occurs within this process, named "inflammaging" [3]. Even if a lot of studies have demonstrated PTS anti-inflammatory activity in different model systems, no study investigated the antiinflammatory effect of PTS in D. melanogaster. On this basis, female and male flies were supplemented with $100 \mu \mathrm{M}$ PTS for 15 days (PTS1) or 60 days (PTS2) and the expression of two pro-inflammatory genes, domeless (dome) and eiger (egr), were measured by RT-PCR (Figure 9). In particular, dome is a signal-transducing receptor with most similarities to the IL-6 receptor family [50], whereas egr is the fly ortho$\log$ of TNF $\alpha$ [51]. In female flies, PTS significantly reduced the expression of dome at 60 days supplementation and the expression of egr at 15 days supplementation. No effect has been observed in male flies.

3.4. Proteome Changes Induced by PTS Supplementation. To understand why PTS supplementation for 15 days counteracted oxidative stress in male but not in female flies, we decided to look at the proteome changes that may be aroused from this supplementation in the two sexes. Figure 10 shows representative 2DE images of protein extracts of bodies of both female (A) and male (B) flies supplemented with $100 \mu \mathrm{M}$ PTS for 15 days. Comparative 

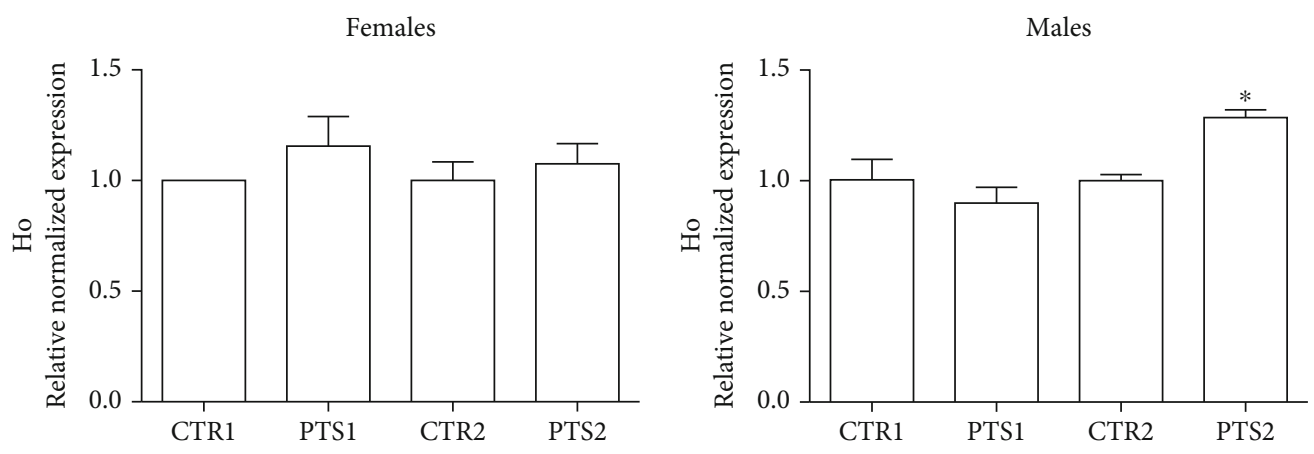

(a)
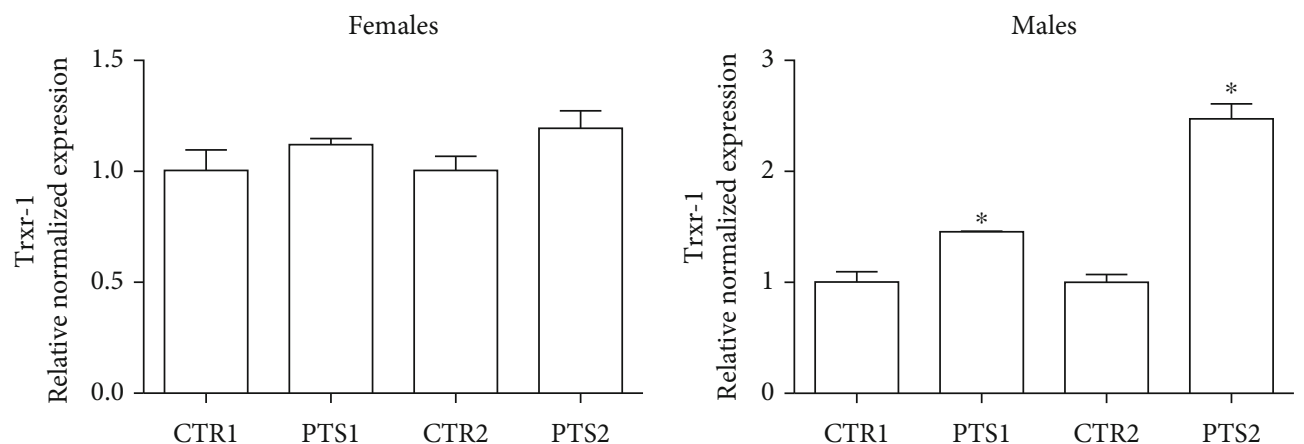

(b)

FIGURE 8: Expression of antioxidants enzymes in D. melanogaster supplemented with PTS. Flies were supplemented with $100 \mu \mathrm{M}$ PTS for 15 days (PTS1) or 60 days (PTS2). Total RNA was isolated, and the mRNA level of A) Ho and B) Trxr-1 was quantified using RT-PCR normalized to RPL32 reference gene as reported in Materials and Methods. Triplicate reactions were performed for each experiment. Each bar represents the mean \pm SEM of three independent experiments. Data relative to 15 days and 60 days were grouped into one graph but analyzed separately by Student's t-test. $* \mathrm{p}<0.05$ with respect to controls, CTR1 and CTR2.

analysis was performed to investigate the effects of PTS in respect to control both in female and male groups. Onehundred and thirteen and nine spots were found differentially expressed (ratio $\geq 1.2$ or $\leq 0.83$, $p<0.05$, q value $<0.05$ ) in male and female flies supplemented with PTS in respect to controls, respectively. For males, only 23 proteins were identified, of which 19 were significantly induced in respect to control flies and 4 were significantly lower than control flies. Venn diagram (Figure 11) illustrates the number of spots differentially expressed which are common and exclusive between the two sexes. Of the nine proteins modulated by PTS in female flies, only two were in common with the proteins differentially expressed in males. Spots of interest were subsequently subjected to nLC-ESI-MS/MS analysis and identified. Table 2 shows the list of identified proteins together with their MW, pI, peptides and coverage values of MS/MS, ratio and $\mathrm{p}$ values. Moreover, normalized mean values of optical density of the identified differentially expressed spots were analyzed using Next-Generation Clustered Heat Map to generate a clustered heat map (Figure 12). In the heat map it is possible to appreciate the higher number of up-regulated proteins after PTS treatment in male flies compared to females. In particular, as suggested by gene ontology analysis, the major changes observed in the males involve proteins belonging to metabolic processes (i.e. small molecule metabolic process, carboxylic acid, oxoacid, ATP, and phosphorus metabolic processes) (Figure 13(b)). Very few changes were observed in the females and essentially related to proteins involved in homeostasis processes and to a lesser degree to metabolic processes (Figure 13(a)).

\section{Discussion}

Recent studies suggest that PTS can be considered a potential candidate as an anti-aging agent thanks to its ability to modulate different hallmarks of aging, including oxidative damage, inflammation, telomere attrition, and cell senescence [52]. The potential health benefits of PTS on lifespan extension are supported by different studies that investigated the effect of blueberry on model organisms. In Caenorhabditis elegans, blueberry polyphenols increased lifespan and thermo-tolerance [6] and blueberry extracts contributed to lifespan prolongation of $D$. melanogaster by the up-regulation of the antioxidant enzymes SOD and catalase [53]. These studies hypothesize that PTS plays a role in increasing longevity but, to the best of our knowledge, no author has so far demonstrated a direct effect of PTS on this parameter. Here, we investigated the effect of PTS supplementation on Drosophila longevity and its potential mechanisms of action.

Our data show that PTS supplementation increases the mean lifespan of male and female flies at specific concentrations. In particular, $100 \mu \mathrm{M}$ PTS was effective in both male and female flies, $50 \mu \mathrm{M}$ increased mean lifespan only in male 

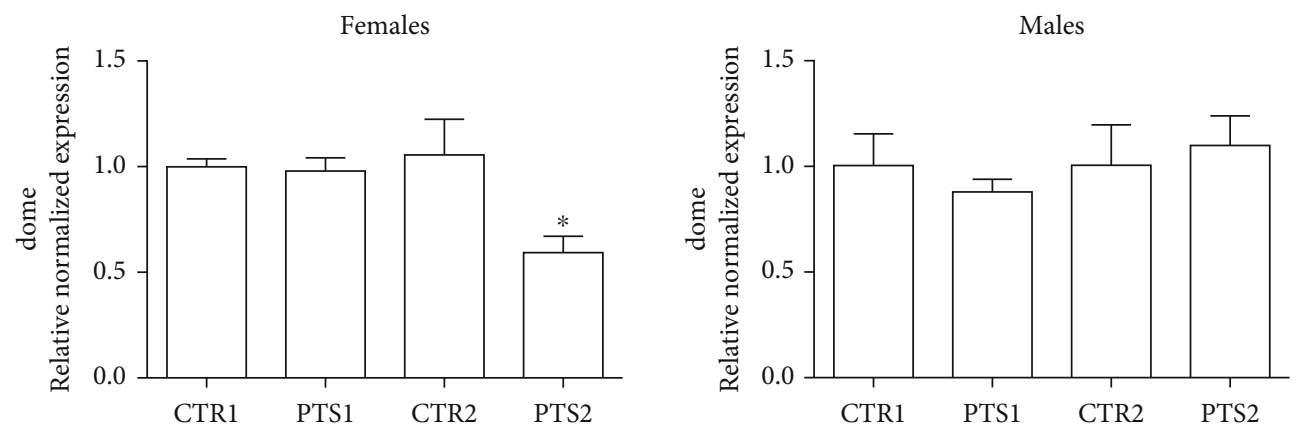

(a)
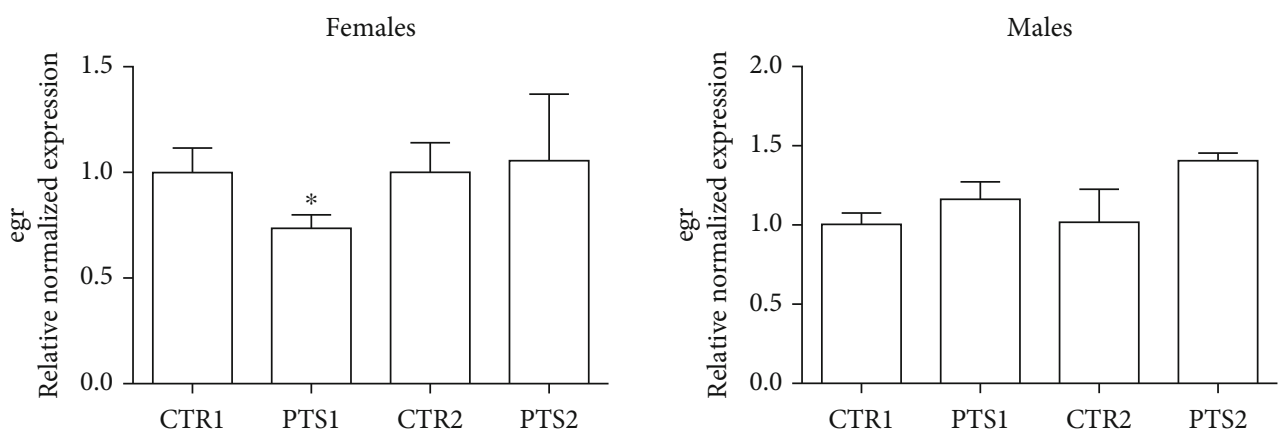

(b)

FIGURE 9: Expression of inflammatory cytokines in D. melanogaster supplemented with PTS. Flies were supplemented with $100 \mu \mathrm{M}$ PTS for 15 days (PTS1) or 60 days (PTS2). Total RNA was isolated, and the mRNA level of A) dome and B) egr was quantified using RT-PCR normalized to RPL32 reference gene as reported in Materials and Methods. Triplicate reactions were performed for each experiment. Each bar represents the mean \pm SEM of three independent experiments. Data relative to 15 days and 60 days were grouped into one graph but analyzed separately by Student's t-test. $* \mathrm{p}<0.05$ with respect to controls, CTR1 and CTR2.

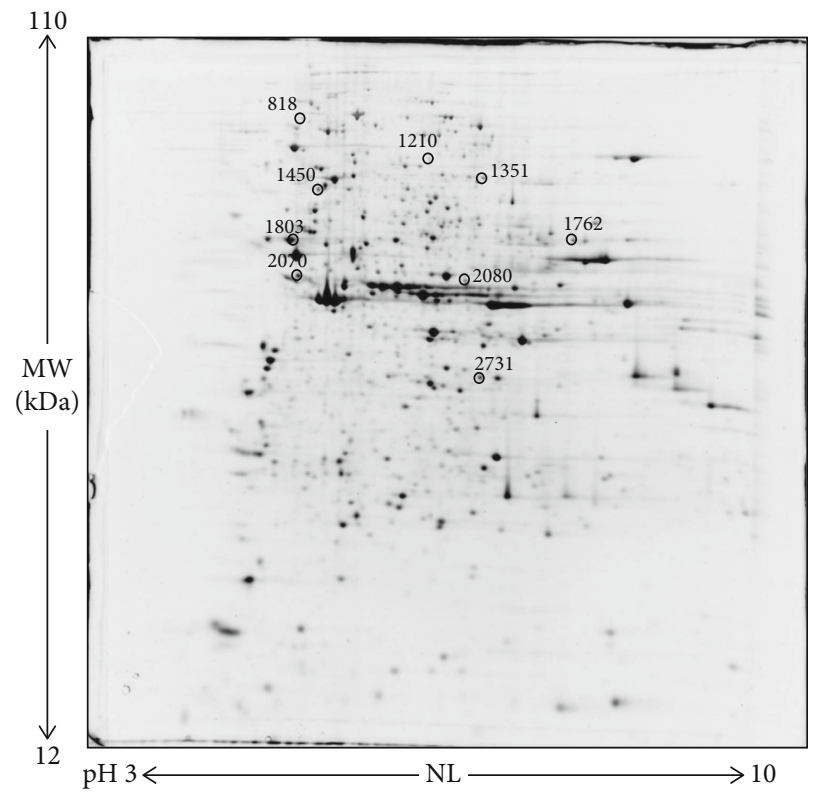

(a)

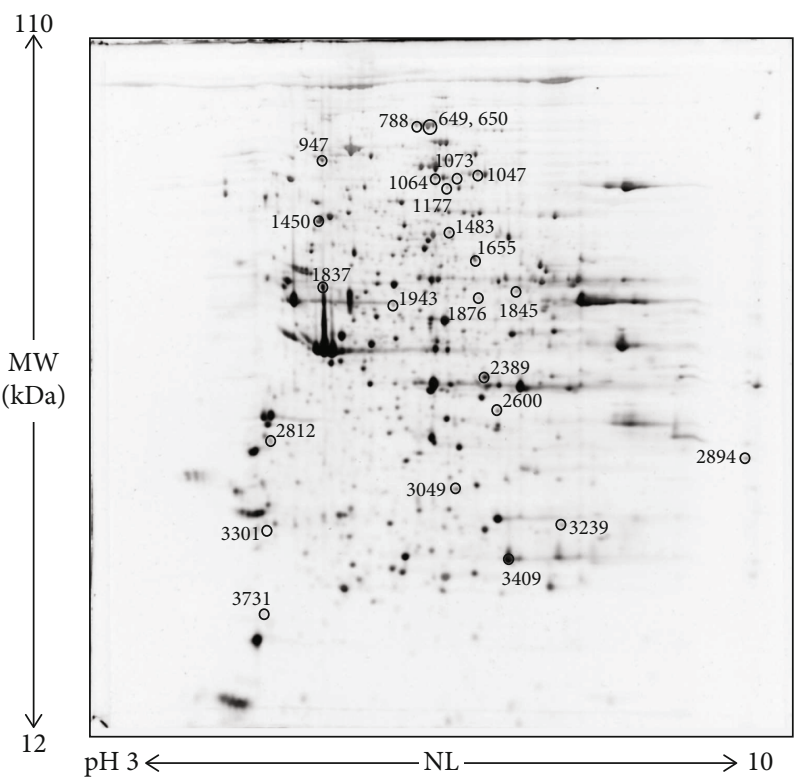

(b)

FIGURE 10: Representative 2DE images of protein extracts of bodies of both female (a) and male (b) flies supplemented with $100 \mu \mathrm{M}$ PTS for 15 days. Protein extracts were separated in a 3-10 nonlinear gradient. SDS-PAGE was performed using 12\% acrylamide. Gels were stained with ruthenium. Spot numbers indicate all the proteins identified by nLC-ESI-MS/MS and refer to the number reported in Table 2. 


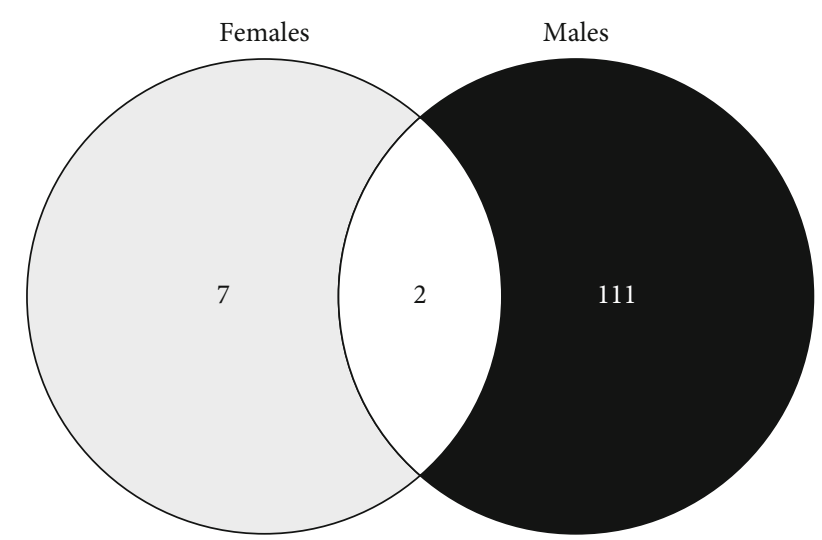

Figure 11: Venn diagram highlighting the distribution of differentially expressed spots in protein extracts obtained by PTS supplemented flies compared to control flies. Both unique and overlapping spots are reported as actual number (Venny 2.0.2).

flies whereas $200 \mu \mathrm{M}$ PTS evidenced a toxic effect on both sexes. Interestingly, PTS supplementation did not modify the amount of food intake in Drosophila, so the observed effect is not due to a potential CR induced by PTS offflavor. Rather, these results could be related to a hormetic effect, a biphasic dose-response mechanism characterized by a low-dose beneficial effect and a high-dose toxic effect [54]. In agreement with our results, different natural products with a pro-longevity effect have been shown to reduce the lifespan of animals when supplemented at high doses [55]. PTS is structurally like resveratrol as both are monomeric stilbenes with the only difference that PTS has two methoxy $\left(-\mathrm{OCH}_{3}\right)$ groups and one hydroxyl $(-\mathrm{OH})$ group, while resveratrol has three $-\mathrm{OH}$ groups (Figure 1). Different studies investigated the effect of resveratrol supplementation on D. melanogaster lifespan with apparently contradictory results. Staats et al. [56] did not observe any effect on lifespan after a $500 \mu \mathrm{M}$ resveratrol supplementation in $\mathrm{D}$. melanogaster, meanwhile Abolaji et al. [57] showed that resveratrol extended lifespan in a dose dependent manner up to a concentration of $60 \mathrm{mg} / \mathrm{kg}$ diet. Interestingly, $120 \mathrm{mg} / \mathrm{kg}$ diet did not modify lifespan when compared to control flies as observed by Staats et al., suggesting that only specific resveratrol concentrations are effective in prolonging lifespan. These data agree with our observations on the effect of PTS on the mean lifespan of $D$. melanogaster where only one dose in females $(100 \mu \mathrm{M})$ and two doses in males (50 and $100 \mu \mathrm{M}$ ) where able to increase this parameter. Moreover, we observed a higher food intake in male flies compared to female flies and this could explain why $50 \mu \mathrm{M}$ PTS supplementation increased mean lifespan of male flies but was not effective on female flies.

As resveratrol has been demonstrated to be effective in increasing lifespan of different model organisms through the up-regulation of Sirt1 [52], we hypothesized that PTS could act on the same molecular target in Drosophila. It has been shown that Sirt1, a (NAD+)-dependent deacetylase, has a role in lifespan extension, stress resistance and apoptosis reduction $[58,59]$. Sirt1 regulates a great number of downstream molecules, including p53, Foxo1, Foxo3,
Foxo4, E2F1, and Notch $[44,60]$. Our data demonstrate that PTS supplementation up-regulates the Drosophila Sirt1 homolog, Sir2, already after 15 days in females, and in both sexes after 60 days. Of note, to our knowledge, no other study demonstrated that PTS increases Sirt1 in healthy individuals undergoing a physiological aging process. In fact, the research demonstrating PTS ability to increase Sirt1 expression have been carried out in different pathological conditions in which Sirt1 resulted down-regulated in respect to healthy individuals.

To further investigate the effect of PTS on the mean lifespan in D. melanogaster we measured the expression of two downstream target of Sir2, Notch and foxo. The Notch signaling pathway is highly conserved among species from Drosophila to humans. It has a fundamental role in adult central nervous system for neural plasticity and triggers neural differentiation during development [61]. Notch is a membrane-bound transcription factor that is released to the nucleus by a two-step cleavage mechanism [62]. The second cleavage is carried out by Presenilin and knockout of Presenilin causes the impairment of synaptic plasticity and memory formation in mice [63]. Moreover, it has been observed an impairment in spatial learning and memory of mice carrying a heterozygous mutation of Notch [64]. Presente et al. [61] observed that D. melanogaster with a selective loss of Notch function in adulthood show a syndrome that includes loss of flight and premature death from unknown causes. In our study, PTS supplementation triggered a significant up-regulation of Notch with the same regulatory pattern of Sir2, suggesting, as previously underlined, a strong crosstalk between these two proteins.

Foxo is a transcription factor acting as downstream effector of insulin signaling and its activity has been observed to be strictly related with stress resistance and lifespan extension in many organisms including humans [65]. In our model system, PTS led to an increase of foxo expression in female flies only after 15 days supplementation and in male flies only after 2-month supplementation, suggesting a potential role in modulating longevity of both sexes, but indicating that PTS can have different effects in male and female flies.

There are many factors that cause aging, among which oxidative stress and inflammation have been demonstrated to play a fundamental role [66]. Oxidative stress is an imbalance between the production and the removal of reactive oxygen species. According to the oxidative stress theory of aging, the functional impairment typical of elderly is connected to the accumulation of structural alterations due to the oxidative damage to macromolecules by ROS [67]. On these bases, we verified if PTS boosts the Drosophila antioxidant system making flies more resistant to oxidative stress. To mimic this condition, we exposed flies to paraquat $[48,49]$ and ethanol $[47,68]$. Interestingly, after exposure to acute ethanol doses, fruit flies show behaviors similar to those observed in humans and mammalian models [69].

A PTS supplementation for 15 days was able to counteract oxidative stress only in male flies, while it was not effective in female flies, evidencing, once again, the different 


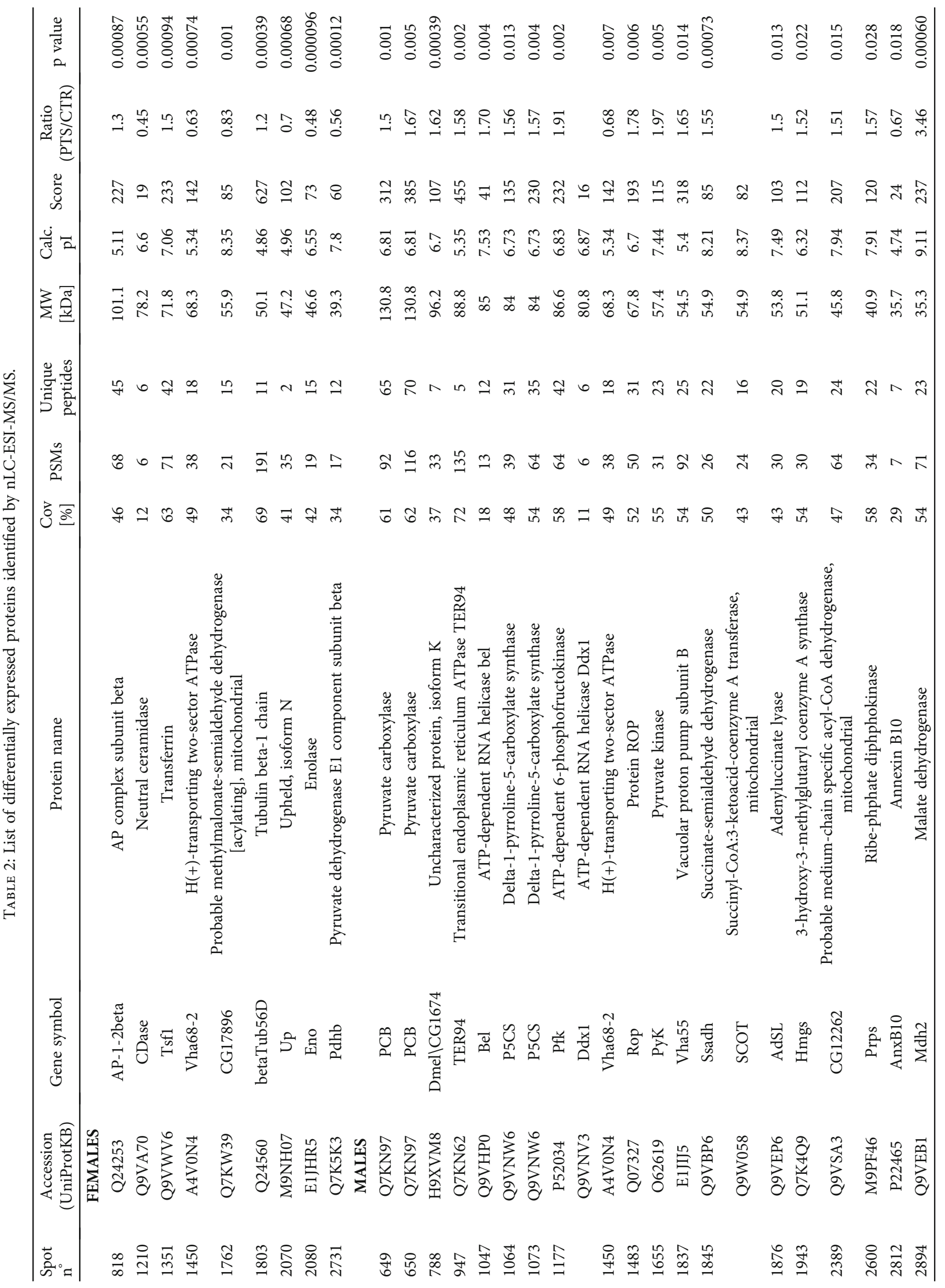




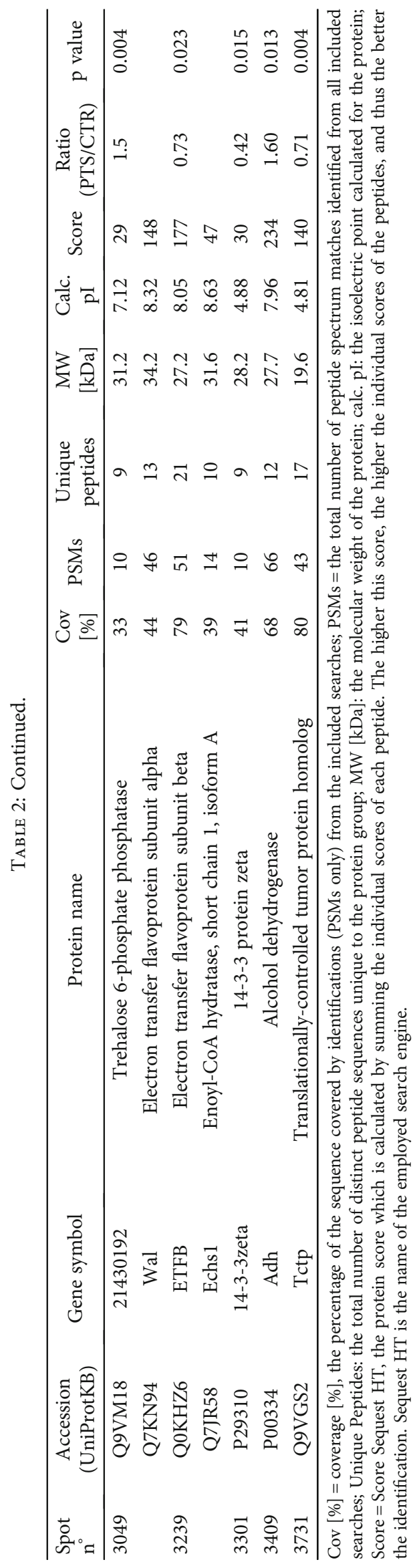




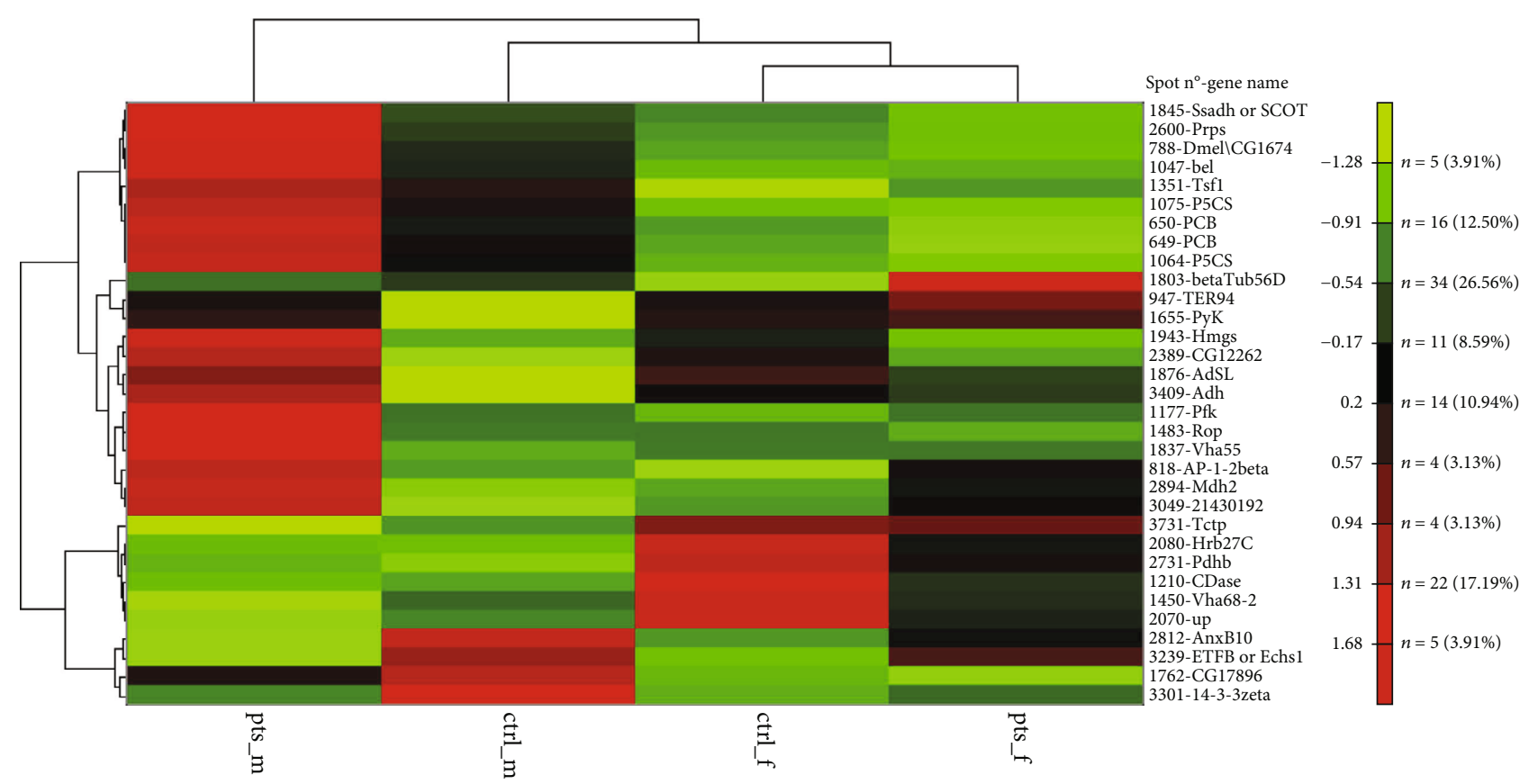

Figure 12: Clustered heat map of differentially expressed proteins after PTS supplementation in female and male samples. Heat map was generated using NG-CHM GUI 2.20.2 software. Z-norm transform was used to normalize the row values (mean of normalized optical densities of spots, $\mathrm{n}=3$ ) and resulting transform data matrix used to build the heat map. A hierarchical ordering method was applied to clustered rows and columns. Euclidean distance metric was applied to hierarchically clustered rows and columns. Data matrix distribution values range from -1.72 to +1.72 . The brighter the color, the more intense the changes are. ctrl_m: male control; ctrl_f: female control; pts_m: males treated with PTS; pts_f: females treated with PTS.

effect of PTS on the two sexes. Interestingly, our data obtained by proteomic analysis indicate that PTS is able to significantly increase alcohol dehydrogenase (ADH) expression only in male flies, supporting the positive PTS activity against ethanol in male flies but not in female flies. In D. melanogaster, $\mathrm{ADH}$ metabolizes more than $90 \%$ of ethanol and in $\mathrm{ADH}$ deficient flies a higher toxicity of ethanol has been observed [70]. Of note, in the first $24 \mathrm{~h}$, paraquat induced a higher mortality in male flies in respect to female flies $(p<0.0063)$. Our results are in agreement with the data of Krůček et al. [71] that observed a higher mortality induced by $25 \mathrm{mM}$ Paraquat in male flies in respect to female flies. This could be ascribed to a different expression of the endogenous antioxidant enzymes in the two sexes. In fact, as demonstrated by Niveditha et al. [33], in female flies the activity of the antioxidant enzymes SOD and catalase is significantly higher in respect to male flies. These data are in agreement with our results that evidenced a higher antioxidant capacity of females flies $(\sim 3.9 \mathrm{mg} \mathrm{TE} / \mathrm{mg}$ protein) in respect to male flies ( $\sim 1.7 \mathrm{mg} \mathrm{TE} / \mathrm{mg}$ protein).

To have a better insight on the antioxidant mechanisms of PTS in Drosophila we investigated the effect of PTS on the expression of two antioxidants enzymes, Ho and Trxr-1. We decided to investigate these two antioxidant enzymes because Ho has been demonstrated to be upregulated by PTS in both cell cultures and animals [72-76] and many studies observed an up-regulation of Trxr-1 and Trx-1 triggered by resveratrol $[77,78]$ but no studies investigated PTS effect on Trxr-1. Moreover, Ho and Trxr-1 are classified as vitagenes, a group of genes which are strictly involved in preserving cellular homeostasis during stressful conditions [79]. The vitagene family is composed of the heat shock proteins (Hsp) Hsp32 (also known as HO-1), Hsp70, sirtuins and by the thioredoxin system [70, 80-82]. In agreement with the data on the protection against oxidative stress, PTS up-regulated Ho and Trxr-1 only in male flies. Our hypothesis is that PTS is more effective in inducing the antioxidant system in males in respect to females because the female endogenous antioxidant system is already highly expressed, and maybe PTS is not able to trigger a further increase.

Many studies have associated an anti-inflammatory activity to PTS. It has been described that PTS inhibited mitogen-activated protein kinase (MAPK) phosphorylation and the production of pro-inflammatory cytokines (Interleukin- 6 and TNF- $\alpha$ ) in mouse microglial cells activated by lipopolysaccharides [83]. Moreover, PTS showed inhibitory effect on inflammatory responses following the interaction of 3 T3-L1 adipocytes with RAW 264.7 macrophages [84]. Our data demonstrated that PTS supplementation reduced the expression of two pro-inflammatory cytokines, in particular dome, that has similarities with the mammalian IL-6 receptor family [9] and egr, the fly orthologue of TNF $\alpha$ [51], in female flies, but had no effect in male flies.

We performed proteomic analyses of male and female flies supplemented for 15 days with PTS to better characterized the mechanisms behind the higher protection of PTS against oxidative stress in males in respect to female flies. 


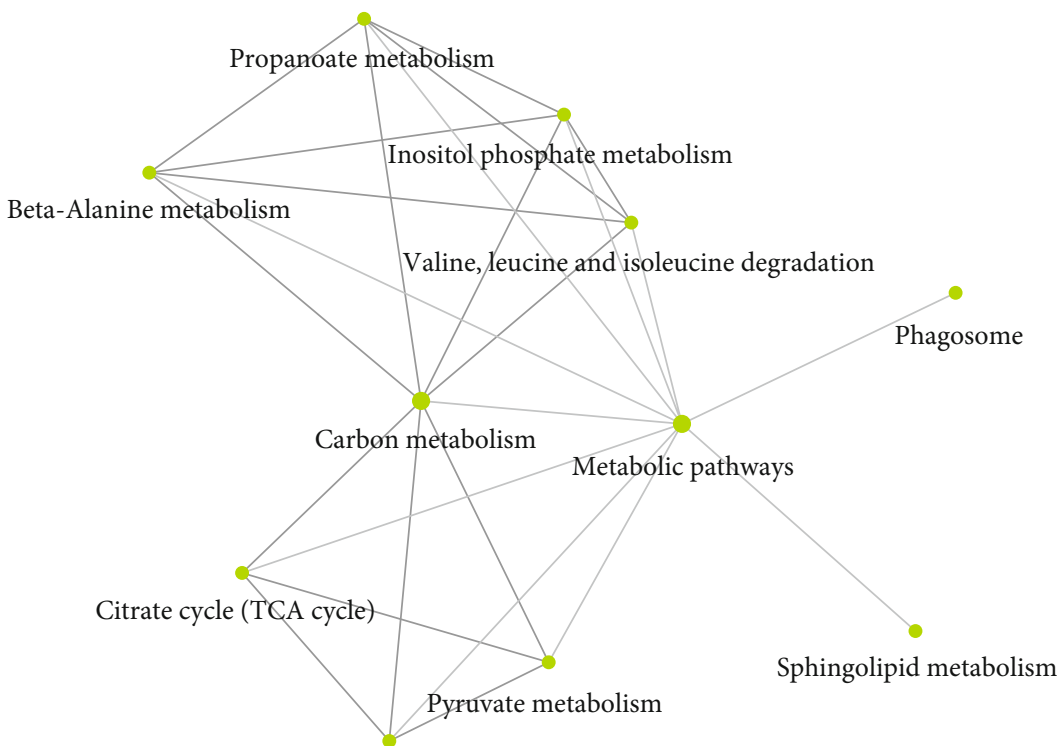

Glycolysis/gluconeogenesis

(a)

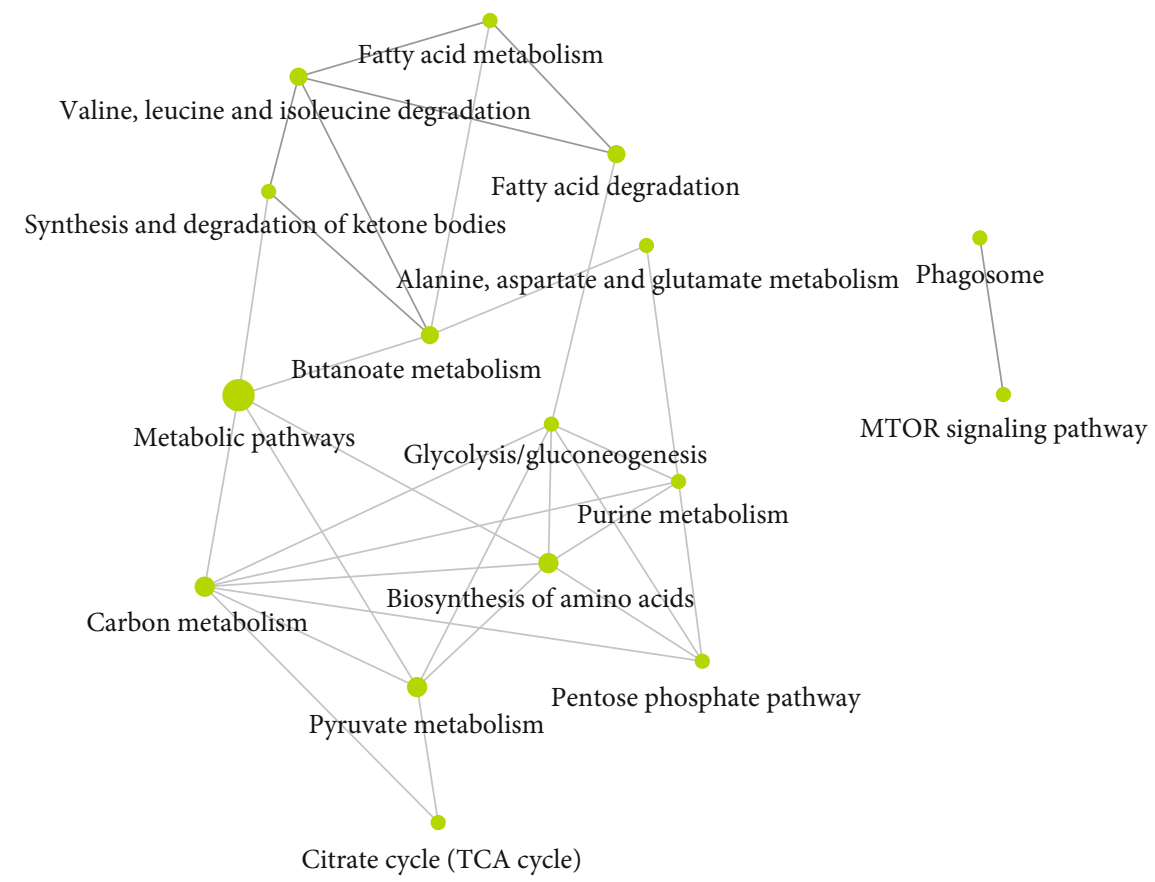

(b)

Figure 13: KEGG Pathway analysis of differentially expressed proteins both in females (a) and males (b) D. melanogaster. ShinyGO v.0.66 software was used to obtain interactive plots which show the relationship between enriched pathways. Two pathways (nodes) are connected if they share $20 \%$ (default) or more genes. Darker nodes are more significantly enriched gene sets. Bigger nodes represent larger gene sets. Thicker edges represent more overlapped genes.

Unlike what we would have expected, the proteomics data did not provide further information useful for understanding the different effect of PTS supplementation on oxidative stress. Nevertheless, several interesting aspects emerged. The results showed that PTS modulates a higher number of proteins in male in respect to female flies (113 and 9, respectively). Among the identified proteins, only one is in common between the two sexes: vacuolar $\mathrm{H}[+]$ ATPase $68 \mathrm{kDa}$ subunit 2 (Vha68-2).The strong down-regulation of Vha682 by PTS is comparable in the two sexes. This protein is part of the vacuolar proton-translocating ATPase (V-ATPase) that is organized into 2 subcomplexes, namely, the ATPcatalyzing domain (V1) and a proton-translocation domain (V0) and Vha68-2 belongs to V0 domain. V-ATPase is a key regulator of organelle acidification in eukaryotic cells $[85,86]$ and has been demonstrated to modulate several 
cellular processes, like membrane trafficking and protein degradation [86, 87]. In D. melanogaster the role of Vha682 has been marginally investigated in only one study related to the function of Drosophila salivary glands during the earlyto mid-prepupal period [88]. However, a role of V-ATPase in longevity has been demonstrated in C. elegans where the RNAi downregulation of 2 different subunits of the $\mathrm{V}$ ATPase, vha- 3 and vha- 12 belonging to $\mathrm{V} 0$ and $\mathrm{V} 1$, respectively, increased lifespan. The authors suggested that this lifespan extension might be due to Ce.TOR inhibition, because $\mathrm{V}$-ATPase is required for the spatial regulation and subsequent activation of TORC1 [89]. On the other hand, only in male flies, vacuolar proton pump subunit B (Vha55), that corresponds to vha-21 in C. elegans [90], is induced by PTS supplementation. These observations suggest that Vha68-2 downregulation by PTS could be involved in the observed increase in both male and female lifespan. Further studies should be carried out to better investigate this point and to verify how this protein is modulated in older flies.

Among the proteins modulated by PTS in female flies, enolase and pyruvate dehydrogenase are particularly interesting as are enzymes related to glucose metabolism, the first one is a glycolytic enzyme, meanwhile the second one transforms the product of glycolysis, pyruvate, in acetyl CoA. Both are strongly downregulated, and this is in agreement with the observations that caloric restriction is characterized by a decreased glucose metabolism [91, 92]. Moreover, Schriner et al. [91], studying the mechanisms behind Rhodiola rosea ability to extend life span in Drosophila, evidenced that $R$. rosea significantly reduced the expression of enolase in female and not in male flies and associated this effect with lifespan increase. This is supported by our data as we observed the downregulation of this enzyme only in female flies that showed a higher mean lifespan in respect to male flies. The limitation of our results is that they were obtained analyzing relatively young flies (15 days old) and as underlined previously, to draw more reliable conclusions, further studies should be carried out to investigate the proteome of older flies.

Among the proteins modulated by PTS in male flies, malate dehydrogenase 2 (mdh2) shows the highest upregulation (fold $>3$ ). Mdh2 is closely related to mitochondrial malate dehydrogenases from multiple animal species, including mouse Mdh2 and yeast Mdh1 and localizes to mitochondria in vivo [93]. Its main role is to transform malate to oxalacetate in the tricarboxylic acid cycle and is critical for cellular energy production. This is supported by the observation of Wang et al. [93] that observed significantly lower levels of ATP in Mdh2 Drosophila mutants and an accumulation of late-stage citric acid cycle intermediates. Of note, this protein is strongly up-regulated in male and not in female flies and this suggests that this up-regulation triggered by PTS could be linked to the presence of specific male hormones. In general, PTS induces different proteins related to metabolic pathways, among them pyruvate carboxylase and kinase, succinate-semialdehyde dehydrogenase, 3hydroxy-3-methylglutaryl coenzyme A synthase, and ATPdependent 6-phosphofructokinase. On the other hand, PTS downregulates few proteins, among them Vha68-2, previously discussed, Annexin B10 (AnxB10), electron transfer flavoprotein subunit beta (ETFB), 14-3-3 protein zeta (14-33zeta), and translationally-controlled tumor protein homo$\log$ (Tctp). Interestingly, in D. melanogaster it has been demonstrated that 14-3-3 genes show strong genetic interaction with Tctp [94], a protein involved in Tor signalling [94-97]. In particular, in Drosophila, Tctp is essential for organ growth by promoting Rheb function for Tor signalling as a guanine nucleotide exchange factor [95]. Moreover, Tctp is over-expressed in cancer cells, and its downregulation induces the reversion of tumour phenotypes [98-100]. From this point of view, the reduction of Tcpt triggered by PTS supplementation in male flies could have a role in counteracting tumorigenesis. Further studies should be carried out to better clarify this interesting aspect.

\section{Conclusions}

In conclusion, our data demonstrate for the first time that PTS increases average lifespan of both male and female flies. Interestingly, the mechanisms behind this effect are different in the two sexes. Protein related to longevity are modulated with different time patterns in the two sexes, moreover PTS was able to increase the expression of two genes (Ho and Trxr-1) involved in the antioxidant defense only in male flies and reduces pro-inflammatory proteins only in female flies. Proteomic analysis suggests a potential involvement of Vha68-2 in the observed increase of lifespan, a reduction of glucose metabolism in female flies and an induction of different metabolic pathways in male flies together with the downregulation of 14-3 3zeta and Tctp suggesting a potential role of PTS in reducing tumorigenesis. These data show that males and females respond differently to treatments, reinforcing the emerging idea that studies on drugs and nutraceuticals should be conducted separately in the two sexes to give more reliable answers. Moreover, these results stressed on the importance of creating personalized treatment, that take into consideration the genetic and biological diversity that arise among different individuals and among the two sexes.

\section{Data Availability}

The data used to support the findings of this study are available from the corresponding author upon request.

\section{Conflicts of Interest}

The authors declare that no conflict of interest and no competing financial interest exist.

\section{References}

[1] D. Harman, "Aging: a theory based on free radicals and radiation chemistry," Journal of Gerontology, vol. 11, no. 3, pp. 298-300, 1956.

[2] L. C. D. Pomatto and K. J. A. Davies, "Adaptive homeostasis and the free radical theory of ageing," Free Radical Biology and Medicine, vol. 124, pp. 420-430, 2018. 
[3] C. Franceschi, M. Bonafè, S. Valensin et al., "Inflamm-aging: An Evolutionary Perspective on Immunosenescence," Annals of the New York Academy of Sciences, vol. 908, pp. 244-254, 2000.

[4] C. Peng, H. Y. Chan, Y. Huang, H. Yu, and Z. Y. Chen, "Apple polyphenols extend the mean lifespan of Drosophila melanogaster," Journal of Agricultural and Food Chemistry, vol. 59, no. 5, pp. 2097-2106, 2011.

[5] T. E. Lopez, H. M. Pham, B. V. Nguyen et al., "Green tea polyphenols require the mitochondrial iron transporter,mitoferrin, for lifespan extension inDrosophila melanogaster," Archives of Insect Biochemistry and Physiology, vol. 93, no. 4, pp. 210-221, 2016.

[6] M. A. Wilson, B. Shukitt-Hale, W. Kalt, D. K. Ingram, J. A. Joseph, and C. A. Wolkow, "Blueberry polyphenols increase lifespan and thermotolerance in Caenorhabditis elegans," Aging Cell, vol. 5, no. 1, pp. 59-68, 2006.

[7] L. Valentini, A. Pinto, I. Bourdel-Marchasson et al., "Impact of personalized diet and probiotic supplementation on inflammation, nutritional parameters and intestinal microbiota - The "RISTOMED project": Randomized controlled trial in healthy older people," Clinical Nutrition, vol. 34, no. 4, pp. 593-602, 2015.

[8] E. Y. Platonova, M. V. Shaposhnikov, H. Y. Lee, J. H. Lee, K. J. Min, and A. Moskalev, "Black chokeberry (Aronia melanocarpa) extracts in terms of geroprotector criteria," Trends in Food Science \& Technology, vol. 114, pp. 570-584, 2021.

[9] K. S. Bhullar and B. P. Hubbard, "Lifespan and healthspan extension by resveratrol," Biochimica et Biophysica Acta, vol. 1852, no. 6, pp. 1209-1218, 2015.

[10] S. Timmers, J. Auwerx, and P. Schrauwen, "The journey of resveratrol from yeast to human," Aging, vol. 4, no. 3, pp. 146-158, 2012.

[11] J. Chang, A. Rimando, M. Pallas et al., "Low-dose pterostilbene, but not resveratrol, is a potent neuromodulator in aging and Alzheimer's disease," Neurobiology of Aging, vol. 33, no. 9, pp. 2062-2071, 2012.

[12] H. P. Chang, C. C. Lu, J. H. Chiang et al., "Pterostilbene modulates the suppression of multidrug resistance protein 1 and triggers autophagic and apoptotic mechanisms in cisplatinresistant human oral cancer CAR cells via AKT signaling," International Journal of Oncology, vol. 52, no. 5, pp. 15041514, 2018.

[13] P. Rodríguez-Bonilla, L. Méndez-Cazorla, J. M. López-Nicolás, and F. García-Carmona, "Kinetic mechanism and product characterization of the enzymatic peroxidation of pterostilbene as model of the detoxification process of stilbene-type phytoalexins," Phytochemistry, vol. 72, no. 1, pp. 100-108, 2011.

[14] M. Briguglio, S. Hrelia, M. Malaguti et al., "Food Bioactive Compounds and Their Interference in Drug Pharmacokinetic/Pharmacodynamic Profiles," Pharmaceutics, vol. 10, no. 4, p. 277, 2018.

[15] Y. S. Chiou, M. L. Tsai, K. Nagabhushanam et al., "Pterostilbene is more potent than resveratrol in preventing azoxymethane (AOM)-induced colon tumorigenesis via activation of the NF-E2-related factor 2 (Nrf2)-mediated antioxidant signaling Pathway," Journal of Agricultural and Food Chemistry, vol. 59, no. 6, pp. 2725-2733, 2011.

[16] W. Nutakul, H. S. Sobers, P. Qiu et al., "Inhibitory effects of resveratrol and pterostilbene on human colon cancer cells: a side-by-side Comparison," Journal of Agricultural and Food Chemistry, vol. 59, no. 20, pp. 10964-10970, 2011.

[17] K. Hagiwara, N. Kosaka, Y. Yoshioka, R. U. Takahashi, F. Takeshita, and T. Ochiya, "Stilbene derivatives promote Ago2-dependent tumour-suppressive microRNA activity," Scientific Reports, vol. 2, no. 1, p. 314, 2012.

[18] I. M. Kapetanovic, M. Muzzio, Z. Huang, T. N. Thompson, and D. L. McCormick, "Pharmacokinetics, oral bioavailability, and metabolic profile of resveratrol and its dimethylether analog, pterostilbene, in rats," Cancer Chemotherapy and Pharmacology, vol. 68, no. 3, pp. 593-601, 2011.

[19] H. S. Lin, B. D. Yue, and P. C. Ho, "Determination of pterostilbene in rat plasma by a simple HPLC-UV method and its application in pre-clinical pharmacokinetic study," Biomedical Chromatography, vol. 23, no. 12, pp. 1308-1315, 2009.

[20] D. M. Riche, M. E. CL, K. D. Riche et al., "Analysis of safety from a human clinical trial with pterostilbene," Journal of Toxicology, vol. 2013, Article ID 463595, 5 pages, 2013.

[21] P. Wang and S. Sang, "Metabolism and pharmacokinetics of resveratrol and pterostilbene," BioFactors, vol. 44, no. 1, pp. 16-25, 2018.

[22] K. T. Howitz, K. J. Bitterman, H. Y. Cohen et al., "Small molecule activators of sirtuins extend Saccharomyces cerevisiae lifespan," Nature, vol. 425, no. 6954, pp. 191-196, 2003.

[23] B. Agarwal and J. A. Baur, "Resveratrol and life extension," Annals of the New York Academy of Sciences, vol. 1215, no. 1, pp. 138-143, 2011.

[24] C. Burnett, S. Valentini, F. Cabreiro et al., "Absence of effects of Sir2 overexpression on lifespan in C. elegans and Drosophila," Nature, vol. 477, no. 7365, pp. 482-485, 2011.

[25] T. M. Bass, D. Weinkove, K. Houthoofd, D. Gems, and L. Partridge, "Effects of resveratrol on lifespan in Drosophila melanogaster and Caenorhabditis elegans," Mechanisms of Ageing and Development, vol. 128, no. 10, pp. 546-552, 2007.

[26] Y. He and H. Jasper, "Studying aging in Drosophila," Methods, vol. 68, no. 1, pp. 129-133, 2014.

[27] A. Chalkiadaki and L. Guarente, "The multifaceted functions of sirtuins in cancer," Nature Reviews. Cancer, vol. 15, no. 10, pp. 608-624, 2015.

[28] K. G. Ormerod, O. K. LePine, P. S. Abbineni et al., "Drosophila development, physiology, behavior, and lifespan are influenced by altered dietary composition," Fly, vol. 11, no. 3, pp. 153-170, 2017.

[29] N. J. Linford, C. Bilgir, J. Ro, and S. D. Pletcher, "Measurement of lifespan in Drosophila melanogaster," Journal of Visualized Experiments, vol. 71, 2013.

[30] S. Piegholdt, G. Rimbach, and A. E. Wagner, "The phytoestrogen prunetin affects body composition and improves fitness and lifespan in maleDrosophila melanogaster," The FASEB Journal, vol. 30, no. 2, pp. 948-958, 2016.

[31] S. Diegelmann, A. Jansen, S. Jois et al., "The CApillary FEeder Assay Measures Food Intake in Drosophila melanogaster," Journal of Visualized Experiments, vol. 10, no. 121, 2017.

[32] K. Fiocca, M. Barrett, E. A. Waddell et al., "Mannitol ingestion causes concentration-dependent, sex-biased mortality in adults of the fruit fly (Drosophila melanogaster)," PLoS One, vol. 14, no. 5, article e0213760, 2019.

[33] S. Niveditha, S. Deepashree, S. R. Ramesh, and T. Shivanandappa, "Sex differences in oxidative stress resistance in relation to longevity in Drosophila melanogaster," 
Journal of Comparative Physiology. B, vol. 187, no. 7, pp. 899909, 2017.

[34] R. Re, N. Pellegrini, A. Proteggente, A. Pannala, M. Yang, and C. Rice-Evans, "Antioxidant activity applying an improved ABTS radical cation decolorization assay," Free Radical Biology \& Medicine, vol. 26, no. 9-10, pp. 1231-1237, 1999.

[35] M. C. Barbalace, L. Zallocco, D. Beghelli et al., “Antioxidant and Neuroprotective Activity of Extra Virgin Olive Oil Extracts Obtained from Quercetano Cultivar Trees Grown in Different Areas of the Tuscany Region (Italy),," Antioxidants, vol. 10, no. 3, p. 421, 2021.

[36] L. Giusti, C. Angeloni, M. Barbalace et al., "A Proteomic Approach to Uncover Neuroprotective Mechanisms of Oleocanthal against Oxidative Stress," International Journal Of Molecular Sciences, vol. 19, no. 8, p. 2329, 2018.

[37] V. Marzano, S. Pane, G. Foglietta et al., "Mass Spectrometry Based-Proteomic Analysis of Anisakis spp.: A Preliminary Study towards a New Diagnostic Tool," Genes, vol. 11, no. 6, p. 693, 2020.

[38] J. S. Yang, H. J. Nam, M. Seo et al., "OASIS: online application for the survival analysis of lifespan assays performed in aging Research," PLoS One, vol. 6, no. 8, article e23525, 2011.

[39] M. C. Ryan, M. Stucky, C. Wakefield et al., "Interactive clustered heat map builder: an easy web-based tool for creating sophisticated clustered heat maps," F1000Research, vol. 8, 2019.

[40] A. G. Vorobyeva, R. Lee, S. Miller et al., "Cyclopamine Modulates $\gamma$-Secretase-mediated Cleavage of Amyloid Precursor Protein by Altering Its Subcellular Trafficking and Lysosomal Degradation*," The Journal of Biological Chemistry, vol. 289, no. 48, pp. 33258-33274, 2014.

[41] X. Wang, H. Perumalsamy, H. W. Kwon, Y. E. Na, and Y. J. Ahn, "Effects and possible mechanisms of action of acacetin on the behavior and eye morphology of Drosophila models of Alzheimer 's disease," Scientific Reports, vol. 5, no. 1, article 16127, 2015.

[42] X. Wang, J. R. Kim, S. B. Lee et al., "Effects of curcuminoids identified in rhizomes of Curcuma longa on BACE-1 inhibitory and behavioral activity and lifespan of Alzheimer's disease drosophila models," BMC Complementary and Alternative Medicine, vol. 14, no. 1, p. 88, 2014.

[43] S. Frankel, T. Ziafazeli, and B. Rogina, "dSir2 and longevity in Drosophila," Experimental Gerontology, vol. 46, no. 5, pp. 391-396, 2011.

[44] M. Horvath, Z. Mihajlovic, V. Slaninova, R. Perez-Gomez, Y. Moshkin, and A. Krejci, "The silent information regulator 1 (Sirt1) is a positive regulator of the Notch pathway in Drosophila," Biochemical Journal, vol. 473, no. 22, pp. 41294143, 2016.

[45] N. Alic, M. E. Giannakou, I. Papatheodorou et al., "Interplay of dFOXO and two ETS-family transcription factors determines lifespan in Drosophila melanogaster," PLoS Genetics, vol. 10, no. 9, article e1004619, 2014.

[46] S. Deepashree, S. Niveditha, T. Shivanandappa, and S. R. Ramesh, "Oxidative stress resistance as a factor in aging: evidence from an extended longevity phenotype of Drosophila melanogaster," Biogerontology, vol. 20, no. 4, pp. 497-513, 2019.

[47] M. Comporti, C. Signorini, S. Leoncini et al., "Ethanolinduced oxidative stress: basic knowledge," Genes \& Nutrition, vol. 5, no. 2, pp. 101-109, 2010.
[48] M. L. Doran, J. M. Knee, N. Wang et al., "Metabolomic analysis of oxidative stress: Superoxide dismutase mutation and paraquat induced stress in Drosophila melanogaster," Free Radical Biology and Medicine, vol. 113, pp. 323-334, 2017.

[49] R. Hosamani and Muralidhara, "Acute exposure of Drosophila melanogaster to paraquat causes oxidative stress and mitochondrial dysfunction," Archives of Insect Biochemistry and Physiology, vol. 83, no. 1, pp. 25-40, 2013.

[50] S. Brown, N. Hu, and J. C. Hombría, "Identification of the first invertebrate interleukin JAK/STAT receptor, the Drosophila gene domeless," Current Biology, vol. 11, no. 21, pp. 1700-1705, 2001.

[51] J. A. Sanchez, D. Mesquita, M. C. Ingaramo, F. Ariel, M. Milán, and A. Dekanty, "Eiger/TNF $\alpha$-mediated Dilp8 and ROS production coordinate intra-organ growth in Drosophila," PLoS Genetics, vol. 15, no. 8, article e1008133, 2019.

[52] Y. R. Li, S. Li, and C. C. Lin, "Effect of resveratrol and pterostilbene on aging and longevity," BioFactors, vol. 44, no. 1, pp. 69-82, 2018.

[53] C. Peng, Y. Zuo, K. M. Kwan et al., "Blueberry extract prolongs lifespan of Drosophila melanogaster," Experimental Gerontology, vol. 47, no. 2, pp. 170-178, 2012.

[54] S. I. Rattan, "Hormesis in aging," Ageing Research Reviews, vol. 7, no. 1, pp. 63-78, 2008.

[55] S. H. Lee, H. S. An, Y. W. Jung et al., "Korean mistletoe (Viscum album coloratum) extract extends the lifespan of nematodes and fruit flies," Biogerontology, vol. 15, no. 2, pp. 153164, 2014.

[56] S. Staats, A. Wagner, B. Kowalewski et al., "Dietary Resveratrol Does Not Affect Life Span, Body Composition, Stress Response, and Longevity-Related Gene Expression in Drosophila melanogaster," International Journal of Molecular Sciences, vol. 19, no. 1, p. 223, 2018.

[57] A. O. Abolaji, A. O. Adedara, M. A. Adie, M. Vicente-Crespo, and E. O. Farombi, "Resveratrol prolongs lifespan and improves 1-methyl-4-phenyl-1,2,3,6-tetrahydropyridineinduced oxidative damage and behavioural deficits in Drosophila melanogaster," Biochemical and Biophysical Research Communications, vol. 503, no. 2, pp. 1042-1048, 2018.

[58] T. Finkel, C. X. Deng, and R. Mostoslavsky, "Recent progress in the biology and physiology of sirtuins," Nature, vol. 460, no. 7255, pp. 587-591, 2009.

[59] J. Li, L. Feng, Y. Xing et al., "Radioprotective and antioxidant effect of resveratrol in hippocampus by activating Sirtl," International Journal of Molecular Sciences, vol. 15, no. 4, pp. 5928-5939, 2014.

[60] J. Li, C. X. Zhang, Y. M. Liu, K. L. Chen, and G. Chen, “A comparative study of anti-aging properties and mechanism: resveratrol and caloric restriction," Oncotarget, vol. 8, no. 39, pp. 65717-65729, 2017.

[61] A. Presente, A. Andres, and J. S. Nye, "Requirement of notch in adulthood for neurological function and longevity," Neuroreport, vol. 12, no. 15, pp. 3321-3325, 2001.

[62] D. Selkoe and R. Kopan, "NOTCH ANDPRESENILIN: regulated intramembrane proteolysis links development and Degeneration," Annual Review of Neuroscience, vol. 26, no. 1, pp. 565-597, 2003.

[63] C. A. Saura, S. Y. Choi, V. Beglopoulos et al., "Loss of Presenilin Function Causes Impairments of Memory and Synaptic Plasticity Followed by Age-Dependent Neurodegeneration," Neuron, vol. 42, no. 1, pp. 23-36, 2004. 
[64] R. M. Costa, T. Honjo, and A. J. Silva, "Learning and Memory Deficits in Notch Mutant Mice," Current Biology, vol. 13, no. 15, pp. 1348-1354, 2003.

[65] C. J. Kenyon, "The genetics of ageing," Nature, vol. 464, no. 7288, pp. 504-512, 2010.

[66] S. Dodig, I. Čepelak, and I. Pavić, "Hallmarks of senescence and aging," Biochemia Medica, vol. 29, no. 3, article 030501, 2019.

[67] K. B. Beckman and B. N. Ames, "The free radical theory of aging Matures," Physiological Reviews, vol. 78, no. 2, pp. 547-581, 1998.

[68] T. Logan-Garbisch, A. Bortolazzo, P. Luu et al., "Developmental ethanol exposure leads to dysregulation of lipid metabolism and oxidative stress in drosophila," G3: Genes, Genomes, Genetics, vol. 5, no. 1, pp. 49-59, 2014.

[69] F. W. Wolf, A. R. Rodan, L. T. Tsai, and U. Heberlein, "HighResolution analysis of ethanol-induced locomotor stimulation inDrosophila," The Journal of Neuroscience, vol. 22, no. 24, pp. 11035-11044, 2002.

[70] V. Calabrese, D. Boyd-Kimball, G. Scapagnini, and D. A. Butterfield, "Nitric oxide and cellular stress response in brain aging and neurodegenerative disorders: the role of vitagenes," In Vivo, vol. 18, no. 3, pp. 245-267, 2004.

[71] T. Krůček, M. Korandová, M. Šerý, R. Frydrychová, and K. Szakosová, "EFFECT of low doses of herbicide paraquat on antioxidant defense INDrosophila," Archives of Insect Biochemistry and Physiology, vol. 88, no. 4, pp. 235-248, 2015.

[72] H. Deng, H. Li, Z. Y. Ho et al., "Pterostilbene's protective effects against photodamage caused by UVA/UVB irradiation," Pharmazie, vol. 73, no. 11, pp. 651-658, 2018.

[73] X. Fan, L. Wang, J. Huang, H. Lv, X. Deng, and X. Ci, "Pterostilbene reduces acetaminophen-induced liver injury by activating the Nrf2 Antioxidative defense system via the AMPK/Akt/GSK3 $\beta$ Pathway," Cellular Physiology and Biochemistry, vol. 49, no. 5, pp. 1943-1958, 2018.

[74] R. Kosuru, V. Kandula, U. Rai, S. Prakash, Z. Xia, and S. Singh, "Pterostilbene Decreases Cardiac Oxidative Stress and Inflammation via Activation of AMPK/Nrf2/HO-1 Pathway in Fructose-Fed Diabetic Rats," Cardiovascular Drugs and Therapy, vol. 32, no. 2, pp. 147-163, 2018.

[75] Z. W. Chen, H. F. Miu, H. P. Wang et al., "Pterostilbene protects against uraemia serum-induced endothelial cell damage via activation of Keap1/Nrf2/HO-1 signaling," International Urology and Nephrology, vol. 50, no. 3, pp. 559-570, 2018.

[76] Y. Yang, C. Fan, B. Wang et al., "Pterostilbene attenuates high glucose-induced oxidative injury in hippocampal neuronal cells by activating nuclear factor erythroid 2-related factor 2," BBA: Molecular Basis of Disease, vol. 1863, no. 4, pp. 827-837, 2017.

[77] R. Lakshmanan, J. Campbell, G. Ukani et al., "Evaluation of dermal tissue regeneration using resveratrol loaded fibrous matrix in a preclinical mouse model of full-thickness ischemic wound," International Journal of Pharmaceutics, vol. 558, pp. 177-186, 2019.

[78] D. Xu, Y. Li, B. Zhang et al., "Resveratrol alleviate hypoxic pulmonary hypertension via anti-inflammation and antioxidant pathways in rats," International Journal of Medical Sciences, vol. 13, no. 12, pp. 942-954, 2016.

[79] V. Calabrese, C. Cornelius, C. Mancuso et al., "Vitagenes, dietary antioxidants and neuroprotection in neurodegenerative diseases," Frontiers in Bioscience, vol. 14, no. 3, pp. 376397, 2009.

[80] C. Mancuso, G. Scapagini, D. Currò et al., "Mitochondrial dysfunction, free radical generation and cellular stress response in neurodegenerative disorders," Frontiers in Bioscience, vol. 12, no. 1, pp. 1107-1123, 2007.

[81] V. Calabrese, E. Guagliano, M. Sapienza et al., "Redox regulation of cellular stress response in aging and neurodegenerative disorders: role of vitagenes," Neurochemical Research, vol. 32, no. 4-5, pp. 757-773, 2007.

[82] V. Calabrese, C. Cornelius, C. Mancuso et al., "Cellular stress response: a novel target for chemoprevention and nutritional neuroprotection in aging, neurodegenerative disorders and Longevity," Neurochemical Research, vol. 33, no. 12, pp. 2444-2471, 2008.

[83] Y. Hou, G. Xie, F. Miao et al., "Pterostilbene attenuates lipopolysaccharide-induced learning and memory impairment possibly via inhibiting microglia activation and protecting neuronal injury in mice," Progress in NeuroPsychopharmacology \& Biological Psychiatry, vol. 54, pp. 92-102, 2014.

[84] C. L. Hsu, Y. J. Lin, C. T. Ho, and G. C. Yen, "The inhibitory effect of pterostilbene on inflammatory responses during the interaction of 3T3-L1 adipocytes and RAW 264.7 macrophages," Journal of Agricultural and Food Chemistry, vol. 61, no. 3, pp. 602-610, 2013.

[85] M. Forgac, "Vacuolar ATPases: rotary proton pumps in physiology and pathophysiology," Nature Reviews. Molecular Cell Biology, vol. 8, no. 11, pp. 917-929, 2007.

[86] K. Cotter, L. Stransky, C. McGuire, and M. Forgac, "Recent Insights into the Structure, Regulation, and Function of the V-ATPases," Trends in Biochemical Sciences, vol. 40, no. 10, pp. 611-622, 2015.

[87] M. E. Maxson and S. Grinstein, "The vacuolar-type $\mathrm{H}^{+}$ATPase at a glance - more than a proton pump," Journal of Cell Science, vol. 127, Part 23, pp. 4987-4993, 2014.

[88] R. Farkaš, D. Beňová-Liszeková, L. Mentelová et al., "Vacuole dynamics in the salivary glands ofDrosophila melanogasterduring prepupal development," Growth \& Differentiation, vol. 57, no. 1, pp. 74-96, 2015.

[89] J. Chen, Y. Ou, Y. Li, S. Hu, L. W. Shao, and Y. Liu, "Metformin extends C. elegans lifespan through lysosomal pathway," eLife, vol. 6, no. 10, p. 13, 2017.

[90] A. K. Allan, J. Du, S. A. Davies, and J. A. Dow, "Genome-wide survey of V-ATPase genes in Drosophila reveals a conserved renal phenotype for lethal alleles," Physiol Genomics, vol. 22, no. 2, pp. 128-138, 2005.

[91] S. E. Schriner, K. Lee, S. Truong et al., "Extension of drosophila lifespan by Rhodiola rosea through a mechanism independent from dietary Restriction," PLoS One, vol. 8, no. 5, article e63886, 2013.

[92] D. K. Ingram and G. S. Roth, "Glycolytic inhibition: an effective strategy for developing calorie restriction mimetics," Geroscience, vol. 43, no. 3, pp. 1159-1169, 2021.

[93] L. Wang, G. Lam, and C. S. Thummel, "Med24andMdh2are required forDrosophilalarval salivary gland cell death," Developmental Dynamics, vol. 239, no. 3, pp. 954-964, 2010.

[94] T. P. Le, L. T. Vuong, A. R. Kim, Y. C. Hsu, and K. W. Choi, "14-3-3 proteins regulate Tctp-Rheb interaction for organ growth in Drosophila," Nature Communications, vol. 7, no. 1, article 11501, 2016. 
[95] Y. C. Hsu, J. J. Chern, Y. Cai, M. Liu, and K. W. Choi, "Drosophila TCTP is essential for growth and proliferation through regulation of dRheb GTPase," Nature, vol. 445, no. 7129, pp. 785-788, 2007.

[96] D. Kobayashi, M. Hirayama, Y. Komohara et al., “Translationally Controlled Tumor Protein Is a Novel Biological Target for Neurofibromatosis Type 1-associated Tumors," Journal of Biological Chemistry, vol. 289, no. 38, pp. 2631426326, 2014.

[97] X. Dong, B. Yang, Y. Li, C. Zhong, and J. Ding, "Molecular Basis of the Acceleration of the GDP-GTP Exchange of Human Ras Homolog Enriched in Brain by Human Translationally Controlled Tumor Protein," Journal of Biological Chemistry, vol. 284, no. 35, pp. 23754-23764, 2009.

[98] M. J. Koziol and J. B. Gurdon, "TCTP in development and cancer," Biochemistry Research International, vol. 2012, Article ID 105203, 9 pages, 2012.

[99] M. Tuynder, L. Susini, S. Prieur et al., "Biological models and genes of tumor reversion: cellular reprogramming through tpt1/TCTP and SIAH-1," Proceedings of the National Academy of Sciences of the United States of America, vol. 99, no. 23, pp. 14976-14981, 2002.

[100] R. Amson, S. Pece, J. C. Marine, P. P. Di Fiore, and A. Telerman, "TPT1/TCTP-regulated pathways in phenotypic reprogramming," Trends in Cell Biology, vol. 23, no. 1, pp. 37-46, 2013. 